\title{
SIX-POINT CONFIGURATIONS IN THE HYPERBOLIC PLANE AND ERGODICITY OF THE MAPPING CLASS GROUP
}

\author{
JULIEN MARCHÉ AND MAXIME WOLFF
}

\begin{abstract}
Let $X$ be the space of isometry classes of ordered sextuples of points in the hyperbolic plane such that the product of the six corresponding rotations of angle $\pi$ is the identity. This space $X$ is closely related to the $\mathrm{PSL}_{2}(\mathbb{R})$-character variety of the genus 2 surface $\Sigma$. In this article we study the topology and the natural symplectic structure on $X$, and we describe the action of the mapping class group of $\Sigma$ on $X$. This completes the classification of the ergodic components of the character variety in genus 2 initiated in [13. MSC Classification: 58D29,
\end{abstract}

57M05, 20H10, 30F60.

\section{InTRODUCTION AND STATEMENTS}

1.1. A simple dynamical system. The hyperbolic plane $\mathbb{H}^{2}$ is naturally identified to the subspace of $\mathrm{PSL}_{2}(\mathbb{R})$ consisting of matrices (up to sign) of trace 0, via the map which associates to a point $x \in \mathbb{H}^{2}$ the rotation $s_{x}$ of angle $\pi$.

In this article we will be studying the following space of configurations of sextuples,

$$
\text { Sex }=\left\{\left(x_{1}, \ldots, x_{6}\right) \in\left(\mathbb{H}^{2}\right)^{6} \mid s_{x_{6}} \cdots s_{x_{1}}=1\right\},
$$

and its quotient $X=\operatorname{Sex} / \mathrm{PSL}_{2}(\mathbb{R})$ by the natural diagonal action.

Given a configuration $\left(x_{1}, \ldots, x_{6}\right) \in \operatorname{Sex}$, and $i \in\{1, \ldots, 6\}$, we may perform a leapfrog move $L_{i}$, which consists in replacing $x_{i}$ with $x_{i+1}$ (in cyclic notation) and $x_{i+1}$ with $s_{x_{i+1}}\left(x_{i}\right)$. In this move, $x_{i}$ "comes to $x_{i+1}$ and pushes it by the same motion": this evokes the leapfrog game played by children, although it may be more accurate to think of a move in Chinese checkers. This move leaves invariant the four other points, as well as the product $s_{x_{i+1}} s_{x_{i}}$, hence preserves Sex. We will denote by $\operatorname{Mod}\left(S_{o}\right)$ the group generated by these leapfrog moves; this notation will become clear later.

As a simple example of configuration $\left(x_{1}, \ldots, x_{6}\right) \in$ Sex we may choose $x_{1}, x_{3}$ and $x_{5}$ arbitrarily and set $x_{2}=x_{1}, x_{4}=x_{3}$ and $x_{6}=x_{5}$. Such a configuration, as well as all the elements of their $\operatorname{Mod}\left(S_{o}\right)$-orbits, will be called pinched configurations. If moreover $x_{1}=x_{3}=x_{5}$ we call it a singular configuration: these configurations yield the only singular point of $X$. We will denote by $X^{*}$ the space of isometry classes of non-singular configurations, and by $X^{\times}$the space of isometry classes of configurations of six points which do not lie in the same geodesic line in $\mathbb{H}^{2}$.

It will be a simple observation that $X$ has three connected components; we will denote by $X_{0}$ the one containing the isometry class of singular configurations. We will prove the following statements:

Theorem 1.1. Let $x \in X_{0}$ be an isometry class of non-pinched configurations. There is a sequence $\left(\gamma_{n}\right)_{n \geq 0}$ in $\operatorname{Mod}\left(S_{o}\right)$ such that the sequence $\left(\left[\gamma_{n} \cdot x\right]\right)_{n \geq 0}$ converges to the isometry class of the singular configurations. The sequence is provided by a geometric algorithm.

Theorem 1.2. The group $\operatorname{Mod}\left(S_{o}\right)$ acts on $X_{0}$ ergodically. 
Along the way, we will show that $X_{0}$ is homeomorphic to a conical neighbourhood of its singularity and derive its homeomorphic type.

The space $X_{0}$, being a real algebraic variety, has a natural (Lebesgue) class of measures for which it makes sense, as in Theorem 1.2, to say that the action of $\operatorname{Mod}\left(S_{o}\right)$ is ergodic. Moreover, $X_{0}^{*}$ also has a natural symplectic structure, related to its interpretation as a character variety.

1.2. Sextuples and representation spaces. Let $\Sigma$ be a genus two surface, let $\Gamma$ denote the fundamental group of $\Sigma$, and let $X(\Gamma)=\operatorname{Hom}\left(\Gamma, \mathrm{PSL}_{2}(\mathbb{R})\right) / \mathrm{PSL}_{2}(\mathbb{R})$ be the space of morphisms of $\Gamma$ in $\mathrm{PSL}_{2}(\mathbb{R})$ up to conjugacy. A representation $\rho: \Gamma \rightarrow \mathrm{PSL}_{2}(\mathbb{R})$ is called elementary if it has a finite orbit in $\overline{\mathbb{H}^{2}}$. Equivalently, $\rho$ is non-elementary if its image is Zariski-dense in $\mathrm{PSL}_{2}(\mathbb{R})$. We denote by $X^{\times}(\Gamma)$ the space of conjugacy classes of non-elementary representations. By work of W. Goldman [7] this is a smooth 6-dimensional symplectic manifold.

Let $\operatorname{Mod}(\Sigma)$ be the mapping class group of $\Sigma$. By the Dehn-Nielsen-Baer theorem, this group may be viewed as the quotient $\mathrm{Out}^{+}(\Gamma)=\operatorname{Aut}^{+}(\Gamma) / \operatorname{Inn}(\Gamma)$ of orientation-preserving automorphisms of $\Gamma$ up to inner automorphisms. A class $[\varphi] \in \operatorname{Out}^{+}(\Gamma)$ acts on a conjugacy class $[\rho] \in X(\Gamma)$ by the formula $[\varphi] \cdot[\rho]=\left[\rho \circ \varphi^{-1}\right]$. In genus two, $\operatorname{Mod}(\Sigma)$ has a special element, the hyperelliptic involution, which generates its center.

The Euler class eu: $X(\Gamma) \rightarrow\{-2,-1,0,1,2\}$ measures the obstruction of lifting the representations $\Gamma \rightarrow \mathrm{PSL}_{2}(\mathbb{R})$ to the universal cover $\widetilde{\mathrm{PSL}}_{2}(\mathbb{R})$. By work of W. Goldman [8], for each $k \in\{-2,-1,1,2\}$, the set $X_{k}^{\times}(\Gamma)$ of classes of representations of Euler class $k$ is connected, and we proved in [13] that the set $X_{0}^{\times}(\Gamma)$ splits into two disjoint open sets $X_{0}^{+}(\Gamma)$ and $X_{0}^{-}(\Gamma)$, that we denoted by $\mathcal{M}_{+}$and $\mathcal{M}_{-}$. The hyperelliptic involution fixes $X_{0}^{+}(\Gamma)$ pointwise, whereas it acts on $X_{0}^{-}(\Gamma)$ as the conjugation by orientation-reversing isometries of the plane. A consequence of [13], Proposition 1.2, is that both $X_{0}^{+}(\Gamma)$ and $X_{0}^{-}(\Gamma)$ are connected. We will discuss briefly this connectedness in Section 3.3.

If $\varphi \in \operatorname{Diff}_{+}(\Sigma)$ represents the hyperelliptic involution, the quotient $S_{o}$ of $\Sigma$ by the action of $\varphi$ has the structure of a spherical orbifold with six points of order 2 . Let $\Gamma_{o}$ denote its orbifold fundamental group. As $\Gamma_{o}$ has the natural following presentation

$$
\Gamma_{o}=\left\langle c_{1}, c_{2}, c_{3}, c_{4}, c_{5}, c_{6} \mid c_{i}^{2}=1, c_{1} \cdots c_{6}=1\right\rangle,
$$

there is an obvious identification between Sex and the space $\operatorname{Hom}^{\prime}\left(\Gamma_{o}, \mathrm{PSL}_{2}(\mathbb{R})\right)$ of morphisms which do not kill any of the $c_{i}$ 's, hence the space $X$ is in bijection with the character variety $X\left(\Gamma_{o}\right)=\operatorname{Hom}^{\prime}\left(\Gamma_{o}, \mathrm{PSL}_{2}(\mathbb{R})\right) / \mathrm{PSL}_{2}(\mathbb{R})$.

Now if $\pi: \Sigma \rightarrow S_{o}$ is the quotient by the action of $\varphi$, the natural map $\pi_{*}: \Gamma \rightarrow \Gamma_{o}$ induces a map $\pi^{*}: X\left(\Gamma_{o}\right) \rightarrow X(\Gamma)$, which restricts to a canonical identification between $X_{0}^{\times}$and $X_{0}^{+}(\Gamma)$. Furthermore, this identification is equivariant for the action of the $\operatorname{group} \operatorname{Mod}\left(\Gamma_{o}\right)$ of leapfrog moves on $X_{0}^{\times}$, and the action of $\operatorname{Mod}(\Gamma)$ on $X_{0}^{+}(\Gamma)$.

1.3. Dynamics of the mapping class group on $\mathrm{PSL}_{2}(\mathbb{R})$-characters in genus two. In 13 we studied the dynamics of $\operatorname{Mod}(\Sigma)$ on $X^{\times}(\Gamma)$, leaving behind the component $X_{0}^{+}(\Gamma)$. Namely, we proved that $\operatorname{Mod}(\Sigma)$ acts ergodically on each of the components $X_{-1}^{\times}(\Gamma), X_{1}^{\times}(\Gamma)$ and $X_{0}^{-}(\Gamma)$, and proved the related result that every representation in these connected components sends some simple closed curve to a non-hyperbolic element of $\mathrm{PSL}_{2}(\mathbb{R})$. The proof of the ergodicity in [13] is strongly related to the existence of non-separating simple closed curves mapped to non-hyperbolic elements, which we proved for almost every representation in these components. By Proposition 1.2 of [13], the same technique cannot be applied to representations in $X_{0}^{+}(\Gamma)$.

An easy consequence of Theorem 1.1 is that every representation in $X_{0}^{+}(\Gamma)$ sends some separating simple closed curve either to the identity or to an elliptic element of $\mathrm{PSL}_{2}(\mathbb{R})$. Then the 
techniques for proving the ergodicity of $\operatorname{Mod}(\Sigma)$ are more involved than in [13. Together with the results of [13, Theorems 1.1 and 1.2 yield the following statements:

Theorem 1.3. Let $\rho: \Gamma \rightarrow \mathrm{PSL}_{2}(\mathbb{R})$ be a representation mapping every simple closed curve to a hyperbolic element. Then $\rho$ is faithful and discrete.

Theorem 1.4. The mapping class group $\operatorname{Mod}(\Sigma)$ acts ergodically on each connected component of non-extremal Euler class of $X^{\times}(\Gamma)$.

Theorem 1.3 gives an affirmative answer to a question of B. Bowditch (see [1], question C) in the genus two case, while Theorem 1.4 proves a conjecture of W. Goldman in the genus two case.

If a representation $\rho: \Gamma \rightarrow \mathrm{PSL}_{2}(\mathbb{R})$ sends a separating simple curve to an elliptic element, we may think of the restriction of $\rho$ on the fundamental group of each of the two one-holed tori as the holonomy of a conic hyperbolic structure on a torus with one cone point. Thus we may think geometrically of a generic representation in $X_{0}^{+}(\Gamma)$ as two such tori glued along their cone points. For this reason, we like to call hourglass these representations.

1.4. Brief outline of the proofs. The dynamical system of sextuples of points in $\mathbb{H}^{2}$ acted on by leapfrog moves is simple enough to find, for every possible non-pinched configuration, an explicit sequence of leapfrog moves which decreases the sum $\sum_{i=1}^{6} d\left(x_{i}, x_{i+1}\right)$. This is done case by case, and leads to the proof of Theorem 1.1. This also yields a geometric algorithm which, given any non-elementary representation of $\Gamma_{o}$ in $\mathrm{PSL}_{2}(\mathbb{R})$, decides whether it is discrete; thus extending the results of [6] and [5] to the group $\Gamma_{o}$.

Theorem 1.1 enables to reduce the proof of Theorem 1.2 to a neighbourhood of the singular representation as in [3]. This neighbourhood has several natural, simple and useful interpretations. First, as a set of limits of sextuple configurations in $\mathbb{H}^{2}$, it may be thought of as a set of configurations of six points in the Euclidean plane, satisfying extra conditions. Second, following [16] or [7], it can be interpreted in terms of the first cohomology group of $\Gamma_{o}$ in $\mathrm{sl}_{2}(\mathbb{R})$ with coefficients twisted by the adjoint action of the singular representation. This leads to a third interpretation as an open set in the cotangent bundle of the Grassmannian of Lagrangians in the symplectic vector space $H_{1}(\Sigma, \mathbb{R})$. Each of these three models bears a natural symplectic structure, and we prove that the natural symplectic structure on $X_{0}^{+}(\Gamma)$ converges to the relevant natural symplectic form on each model, at the singular representation.

The idea is then to use the Dehn twists along the separating curves which are mapped to elliptic elements. The strategy of the proof, as in [9], is to prove that if $[\rho]$ is sufficiently close to the singular class of representations, the corresponding twist flows are transitive on a neighbourhood of $[\rho]$. In the situation at hand, we do not show whether these twist flows generate the space of all directions around our representations (contrarily to [9] or [13), but by using the third model we prove that their directions generate a completely non-integrable distribution of directions, hence these flows are indeed transitive; this leads to the proof of Theorem 1.2.

1.5. Organisation of the article. We introduce some notation in Section 2 and relate our simple dynamical system to the dynamics of the mapping class group in genus 2. In Section 3 , we prove Theorem 1.3. Section 4 is devoted to the neighborhood of the singular configuration whereas Section 5 contains the proof of Theorem 1.4.

1.6. Acknowledgements. This work was partially supported by the french ANR ModGroup ANR-11-BS01-0020 and SGT ANR-11-BS01-0018. The second author acknowledges support 
from U.S. National Science Foundation grants DMS 1107452, 1107263, 1107367 "RNMS: Geometric Structures and Representation Varieties" (the GEAR Network). We would like to thank Tian Yang and Dick Canary for their kind interest.

\section{Configurations of SeXtuples}

The aim of this section is to expand on the relation, mentionned in the introduction, between the space of sextuple configurations and the $\mathrm{PSL}_{2}(\mathbb{R})$-character variety of the surface of genus two. We will first elaborate on the presentation of the marked groups $\Gamma$ and $\Gamma_{o}$, in order to see the group of leapfrog moves as a mapping class group. It is actually isomorphic to the 6 -strands braid group of the sphere. We will then recall some elementary drawings relating products of half-turns; these reminders will be useful later on. We will then expand on the natural map between $X\left(\Gamma_{o}\right)$ and $X(\Gamma)$, and finally exhibit a complete list of types of sextuple configurations, which will be used in the following section.

2.1. Markings of the groups $\Gamma$ and $\Gamma_{o}$. With suitable markings, the groups $\Gamma$ and $\Gamma_{o}$ admit the following presentations,

$$
\begin{gathered}
\Gamma_{o}=\left\langle c_{1}, c_{2}, c_{3}, c_{4}, c_{5}, c_{6} \mid c_{i}^{2}=1, c_{1} \cdots c_{6}=1\right\rangle, \\
\Gamma=\left\langle a_{1}, b_{1}, a_{2}, b_{2} \mid\left[a_{1}, b_{1}\right]\left[a_{2}, b_{2}\right]=1\right\rangle,
\end{gathered}
$$

and the morphism $\pi_{*}$ associated to the quotient by the hyperelliptic involution is defined as follows:

$$
\pi_{*}\left(a_{1}\right)=c_{1} c_{2}, \quad \pi_{*}\left(b_{1}\right)=c_{3} c_{2}, \quad \pi_{*}\left(a_{2}\right)=c_{4} c_{5}, \quad \pi_{*}\left(b_{2}\right)=c_{6} c_{5} .
$$

Figure 1 is meant to help the reader with the above conventions for presenting the groups $\Gamma$ and $\Gamma_{o}$. It should be noted here that, since the product in a fundamental group uses concatenation of paths, words in these groups are to be read from left to right, and our convention for the commutator here is: $[a, b]=a b a^{-1} b^{-1}$. On the other hand, we prefer to think of $\mathrm{PSL}_{2}(\mathbb{R})$ as

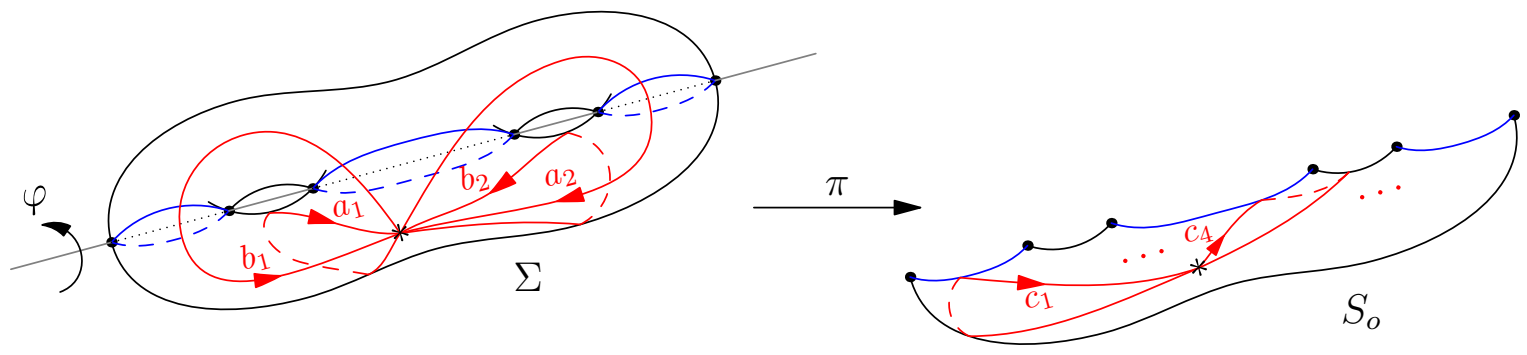

Figure 1. Markings of the groups $\Gamma$ and $\Gamma_{o}$

acting on $\mathbb{H}^{2}$ on the left, hence we prefer to read words in $\mathrm{PSL}_{2}(\mathbb{R})$ from right to left. For this reason, we will take the convention that morphisms $\rho: \Gamma \rightarrow \mathrm{PSL}_{2}(\mathbb{R})$ should be defined as satisfying the relation $\rho(\alpha \beta)=\rho(\beta) \rho(\alpha)$ for all $\alpha, \beta \in \Gamma$. We will also denote, for $A, B \in$ $\mathrm{PSL}_{2}(\mathbb{R}),[A, B]=B^{-1} A^{-1} B A$. This convention is reminiscent of [13] or [4].

Every positive self-diffeomorphism $\psi$ of $\Sigma$ commutes, up to isotopy, with the hyperelliptic involution $\varphi$, hence descends to a diffeomorphism of the sphere with six marked points. This defines an isomorphism between the quotient $\operatorname{Mod}(\Sigma) /[\varphi]$ and the group $B_{6}\left(S^{2}\right)$, the 6 -strands braid group of the sphere. This group is generated by the "standard" generators, often denoted by $\sigma_{i}$, as schematised in Figure 2. As they are depicted in Figure 2 the diffeomorphisms $\sigma_{i}$ fix the base point of $S_{o}$ hence act as automorphisms of $\Gamma_{o}$; we can read: $\sigma_{i *}\left(c_{i}\right)=c_{i+1}$ and 


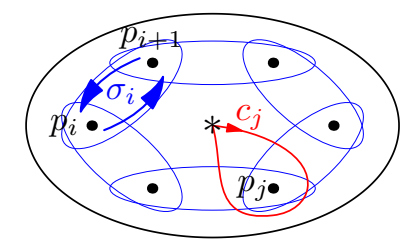

FiguRE 2. Standard generators of $B_{6}\left(S^{2}\right)$

$\sigma_{i *}\left(c_{i+1}\right)=c_{i+1}^{-1} c_{i} c_{i+1}=c_{i+1} c_{i} c_{i+1}$. Hence, the action of $\sigma_{i}$ on representations $\Gamma_{o} \rightarrow \mathrm{PSL}_{2}(\mathbb{R})$ coincides with the action of the leapfrog move $L_{i}$.

In addition to the leapfrog moves corresponding to the $\sigma_{i}$, we will often use the cyclic permutation $\sigma_{5} \sigma_{4} \cdots \sigma_{1}$, which acts on sextuples by permutation, $\left(x_{1}, \ldots, x_{6}\right) \mapsto\left(x_{2}, \ldots, x_{1}\right)$, as well as the "half-twist" $\left(\sigma_{1} \sigma_{2} \sigma_{1}\right)^{2}$, which replaces $c_{1}, c_{2}$ and $c_{3}$ by their conjugates by $c_{1} c_{2} c_{3}$, thus which acts on sextuples by the formula $\left(x_{1}, \ldots, x_{6}\right) \mapsto\left(f\left(x_{1}\right), f\left(x_{2}\right), f\left(x_{3}\right), x_{4}, x_{5}, x_{6}\right)$ where $f=s_{x_{3}} s_{x_{2}} s_{x_{1}}$. We call it this way because it is the image, in $\operatorname{Mod}\left(S_{o}\right)$, of the half-Dehn twist along the separating curve $\left[a_{1}, b_{1}\right]$.

Let us insist, finally, that the $\operatorname{map} \operatorname{Mod}(\Sigma) /[\varphi] \rightarrow B_{6}\left(S^{2}\right)=\operatorname{Mod}\left(S_{o}\right)$ is explicit, and it is easy to translate an explicit sequence of leapfrog moves into an explicit sequence of Dehn twists on $\Sigma$. Namely, in the left part of Figure 1, consider the three blue closed curves, and the two black curves making the two handles of $\Sigma$. It is well-known that the five corresponding Dehn twists generate $\operatorname{Mod}(\Sigma)$. The five Dehn twists along these five curves, ordered from left to right, descend respectively to $\sigma_{1}, \ldots, \sigma_{5}$ in $\operatorname{Mod}\left(S_{o}\right)$.

2.2. Products of three half-turns and commutators. Let $x_{1}, x_{2}, x_{3} \in \mathbb{H}^{2}$ and let $s_{i}, i=$ $1,2,3$, be the half-turn around $x_{i}$. If $x_{1}, x_{2}, x_{3}$ are on a same line, then $s_{1}, s_{2}, s_{3}$ can be thought of as isometries of the real line, and the product $s_{3} s_{2} s_{1}$ is a half-turn around a point easy to spot on this line (see Figure 3, left). If $x_{1}, x_{2}, x_{3}$ are not on a same line, then $x_{3}$ is at some distance $h$ from the line $\left(x_{1}, x_{2}\right)$. Then $s_{3} s_{2} s_{1}=s_{3} s_{2}^{\prime} s_{1}^{\prime}$, where $s_{2}^{\prime}$ is the half-turn around the closest point, $x_{2}^{\prime}$, to $x_{3}$ on the line $\left(x_{1}, x_{2}\right)$, and $s_{1}^{\prime}$ is a half-turn around the point $x_{1}^{\prime} \in\left(x_{1}, x_{2}\right)$ chosen so that $s_{2} s_{1}=s_{2}^{\prime} s_{1}^{\prime}$. Now $s_{3} s_{2} s_{1}$ is the composition of two explicit reflections, $r_{2} r_{1}$ (see Figure 3, right). Depending on whether $\sinh (h) \sinh \left(d\left(x_{1}, x_{2}\right)\right)$ is less, equal or greater than 1 , the hyperbolic
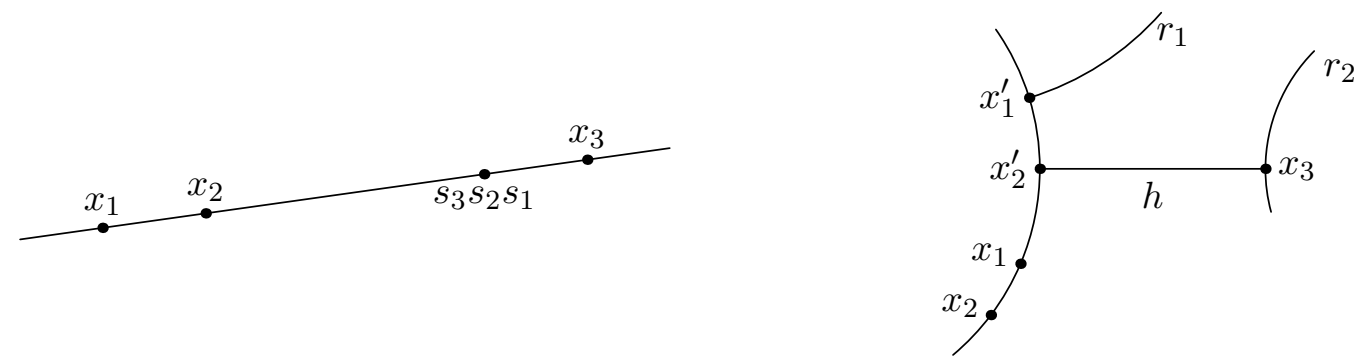

Figure 3. Products of three half-turns (left: the centres are on a line; right: they are in generic position)

motion $s_{3} s_{2} s_{1}$ is elliptic, parabolic or hyperbolic (this follows from the classical formulas in hyperbolic geometry, see eg [2], page 454, formulas 2.3.1 and 2.3.4). It is noteworthy that this quantity $\sinh (h) \sinh \left(d\left(x_{1}, x_{2}\right)\right)$ is equal to $\frac{1}{2} \operatorname{Tr}\left(s_{3} s_{2} s_{1}\right)$ and is invariant under permutations of the $x_{i}^{\prime} s$. It is often called the $\Delta$-invariant of the triangle $x_{1}, x_{2}, x_{3}$. 
This can be applied to describe the geometry of commutators of hyperbolic elements of $\mathrm{PSL}_{2}(\mathbb{R})$ whose axes intersect in $\mathbb{H}^{2}$. If $A, B$ are two such isometries, let $x_{2}$ be the intersection point of these axes (take $x_{2}$ to be any point on this line, if $A$ and $B$ have the same axis). There exists a unique point $x_{1}$ on the axis of $A$ such that, if $s_{1}, s_{2}$ are the half-turns around $x_{1}, x_{2}$ we have $A=s_{2} s_{1}$. Similarly, there exists a unique point $x_{3}$, on the axis of $B$, such that $B=s_{2} s_{3}$, where $s_{3}$ is the half-turn around $x_{3}$. Now,

$$
[A, B]=B^{-1} A^{-1} B A=\left(s_{3} s_{2} s_{1}\right)^{2} .
$$

With this in head, Figure 3 gives a geometric picture of commutators of hyperbolic elements with crossing axes.

2.3. Sextuple configurations as representations of $\Gamma$. The aim of this paragraph is to prove the following correspondence:

Proposition 2.1. The $\mathrm{PSL}_{2}(\mathbb{R})$-equivariant map $\pi^{*}$ : $\operatorname{Sex} \rightarrow \operatorname{Hom}\left(\Gamma, \mathrm{PSL}_{2}(\mathbb{R})\right)$ descends, by restriction, to a homeomorphism $\pi^{*}: X^{\times}\left(\Gamma_{o}\right) \rightarrow X_{-2}(\Gamma) \cup X_{0}^{+}(\Gamma) \cup X_{2}(\Gamma)$.

In the above statement we write "homeomorphism" because we are not yet concerned with the rich structure of these spaces, but of course $\pi^{*}$ carries their structures.

For completeness let us recall the following statement from [13]:

Proposition 2.2 (Part of Proposition 1.2 of [13]). Let $[\rho] \in X_{0}^{+}(\Gamma)$. For every non-separating curve $a, \rho(a)$ is hyperbolic or the identity. For every simple curves $a, b$ such that $i(a, b)=1$, the trace of the commutator $[\rho(a), \rho(b)]$ is in $(-\infty, 2]$. It is 2 if and only if $[\rho(a), \rho(b)]=1$.

Proof of Proposition 2.1. Let $z=\left(x_{1}, \ldots, x_{6}\right) \in$ Sex, and let $\rho=\pi^{*}(z)$. From Equation (11) and the equality $s_{x_{6}} \cdots s_{x_{1}}=1$ we compute, formally,

$$
\rho\left(\left[a_{1}, b_{1}\right]\left[a_{2}, b_{2}\right]\right)=\left(s_{x_{6}} s_{x_{5}} s_{x_{4}}\right)^{2}\left(s_{x_{3}} s_{x_{2}} s_{x_{1}}\right)^{2}=\left(s_{x_{6}} \cdots s_{x_{1}}\right)^{2} .
$$

Moreover, the square enables to lift $\rho$ to a representation in $\mathrm{SL}_{2}(\mathbb{R})$; this means that $\rho$ has even Euler class. Now the representation of $\Gamma_{o}$ defined by $z$ is elementary if and only if $x_{1}, \ldots, x_{6}$ are on the same line, and restricting a representation to an index two subgroup does not change elementarity. This implies that the image of $X^{\times}\left(\Gamma^{o}\right)$ is contained in $X_{-2}(\Gamma) \cup X_{0}^{+}(\Gamma) \cup X_{2}(\Gamma)$, because, by its construction, the conjugacy class of $\rho$ is invariant under the hyperelliptic involution.

Conversely, let $[\rho] \in X_{-2}(\Gamma) \cup X_{0}^{+}(\Gamma) \cup X_{2}(\Gamma)$. Suppose for simplicity that $\left[\rho\left(a_{1}\right), \rho\left(b_{1}\right)\right] \neq 1$. Recall that for $A, B \in \mathrm{PSL}_{2}(\mathbb{R})$, the trace of $[A, B]$ is well defined, and it is in $(-\infty, 2]$ if and only if $A, B$ are hyperbolic and their axes cross each other. Then $\rho\left(a_{1}\right)$ and $\rho\left(b_{1}\right)$ are hyperbolic with crossing axes; they define three points $x_{1}, x_{2}$ and $x_{3}$ exactly as in the last paragraph of Section 2.2. Similarly, define $x_{4}, x_{5}$ and $x_{6}$ corresponding to $\rho\left(a_{2}\right)$ and $\rho\left(b_{2}\right)$. Then we readily check that $\pi^{*}\left(x_{1}, \ldots, x_{6}\right)=\rho$.

Now if $\left[\rho\left(a_{1}\right), \rho\left(b_{1}\right)\right]=1$, we may (for example) first apply an explicit automorphism $\psi$ of $\Gamma$ to change $\left(a_{1}, b_{1}\right)$ into $(a, b)$ with $a, b \in\left\{a_{1}, b_{1}, a_{2}, b_{2}\right\}$ and $i(a, b)=1$, so that $[\rho(a), \rho(b)] \neq 1$ (otherwise $\rho$ would be elementary), make the above construction, and come back to the original marking by applying $\psi^{-1}$. Thus, we have constructed a map $X_{-2}(\Gamma) \cup X_{0}^{+}(\Gamma) \cup X_{2}(\Gamma) \rightarrow X^{\times}\left(\Gamma_{o}\right)$, and we easily check that this map, and $\pi^{*}$, are the inverse to each other.

2.4. Different types of sextuple configurations. The aim of this paragraph is to have in head a picture of every possible configuration of sextuples $z=\left(x_{1}, \ldots, x_{6}\right) \in$ Sex, and to set up some notation for the use of the following section.

In the defining equality we may group the terms two by two, $\left(s_{x_{6}} s_{x_{5}}\right)\left(s_{x_{4}} s_{x_{3}}\right)\left(s_{x_{2}} s_{x_{1}}\right)=1$. For all $i \in\{1, \ldots, 6\}$ we will write $a_{i, i+1}=d\left(x_{i}, x_{i+1}\right)$, with cyclic notation. If, say, $x_{1}=x_{2}$ 
then the above relation implies that $x_{3}, x_{4}, x_{5}, x_{6}$ are on the same line. In this case, it is easy to deform $z$ among sextuple configurations into a singular configuration. Now we want to describe the generic configurations, in which $a_{i, i+1} \neq 0$ for all $i$. We then denote by $D_{i, i+1}$ the line joining $x_{i}$ and $x_{i+1}$. Note that $s_{i+1} s_{i}$ is a hyperbolic translation along that line.

It is elementary and classical to picture the product of two given hyperbolic motions, say $s_{x_{2}} s_{x_{1}}$ and $s_{x_{4}} s_{x_{3}}$. If their axes $D_{12}$ and $D_{34}$ cross each other in $\mathbb{H}^{2}$, we decompose each of these two motions into two rotations of angle $\pi$, one of them being around their intersection point (as in Paragraph 2.2). The resulting product is the product of the two other half turns. If $D_{12}$ and $D_{34}$ are disjoint in $\mathbb{H}^{2} \cup \partial \mathbb{H}^{2}$ we decompose each of the two motions into two reflections along lines, one of them being the common perpendicular $H_{23}$ of $D_{12}$ and $D_{34}$. Then $s_{x_{6}} s_{x_{5}}$ is the product of the reflections along the two other lines, which, therefore, cannot intersect each other in $\mathbb{H}^{2} \cup \partial \mathbb{H}^{2}$. It follows that the lines $D_{12}, D_{34}$ and $D_{56}$, together with the respective common perpendiculars $H_{23}, H_{45}$ and $H_{61}$, form a right-angled hexagon, which may be regular or skew, depending on whether the directions of the motions $s_{x_{2}} s_{x_{1}}$ and $s_{x_{4}} s_{x_{3}}$ agree or disagree (this makes sense for instance by parallel transport along $H_{23}$ ). The only remaining case is when $D_{12}$ and $D_{34}$ meet at $\partial \mathbb{H}^{2}$; the motions $s_{x_{2}} s_{x_{1}}$ and $s_{x_{4}} s_{x_{3}}$ are then contained in a parabolic subgroup of $\mathrm{PSL}_{2}(\mathbb{R})$. The following lemma summarizes the above discussion.

Lemma 2.3. Let $z=\left(x_{1}, \ldots, x_{6}\right) \in \mathrm{Sex}^{\times}$be a non-aligned configuration with $x_{1} \neq x_{2}, x_{3} \neq x_{4}$ and $x_{5} \neq x_{6}$. Then one of the following holds.

(TRI) The lines $D_{12}, D_{34}$ and $D_{56}$ form a triangle with lengths $a_{12}, a_{34}$ and $a_{56}$.

(PAR) The lines $D_{12}, D_{34}$ and $D_{56}$ meet at (at least) one common point in $\partial \mathbb{H}^{2}$. In this case, one of the lengths $a_{12}, a_{34}$ and $a_{56}$ is the sum of the two others.

(SKH) The lines $D_{12}, D_{34}$ and $D_{56}$ are pairwise disjoint in $\overline{\mathbb{H}^{2}}$ and together with their respective common perpendiculars they form a skewed right-angled hexagon. In this case, one of the lengths $a_{12}, a_{34}$ and $a_{56}$ is greater than the sum of the two others.

(HEX) The lines $D_{12}, D_{34}$ and $D_{56}$ are pairwise disjoint in $\overline{\mathbb{H}^{2}}$ and with their respective common perpendiculars they form a right-angled hexagon.

In each of the three situations (TRI), (PAR) and (SKH) it is easy to deform $z$ among sextuple configurations into a singular configuration; these cases, together with the degenerate cases above and the aligned configurations, form the connected component $X_{0}$. Of course, we will denote by $\operatorname{Sex}_{0}$ the set of corresponding sextuple configurations. Each case comes with two possible orientations (and a choice of repelling or attracting point in case (PAR)). In case (HEX), these two orientations correspond to the two components of $X_{ \pm 2}(\Gamma)$ via Proposition 2.1.

Figures 4 and 5 illustrate the cases (TRI), (PAR) and (SKH) with some extra notation that will be used in the next section. In particular, in every case we will consider the relevant intersection points $y_{1}, \ldots, y_{6}$; they satisfy the relation $s_{x_{i+1}} s_{x_{i}}=s_{y_{i+1}} s_{y_{i}}$ for $i=1,3,5$.

Remark 2.1. In Figure 4 we have drawn the triangular case as a Euclidean one for convenience. It is noteworthy however that if $\mathbb{H}^{2}$ was replaced by $\mathbb{R}^{2}$ we would have an extra degree of freedom: the side lengths of the triangle formed by $D_{12}, D_{34}$ and $D_{56}$ would only be proportional to $a_{12}$, $a_{34}$ and $a_{56}$ by a free positive constant. This phenomenon will have a significant role in Section 4 ,

\section{REDUCTION OF SIZE}

In this section we will first prove that the space $X_{0}$ is contractible, then we will prove Theorem 1.1, we will end this section with miscellaneous considerations. The strategy of both proofs consists in decreasing the relative distances between the points $x_{i}$, but as the considerations of the first proof are more differential and the second more discrete in nature, we will use different 

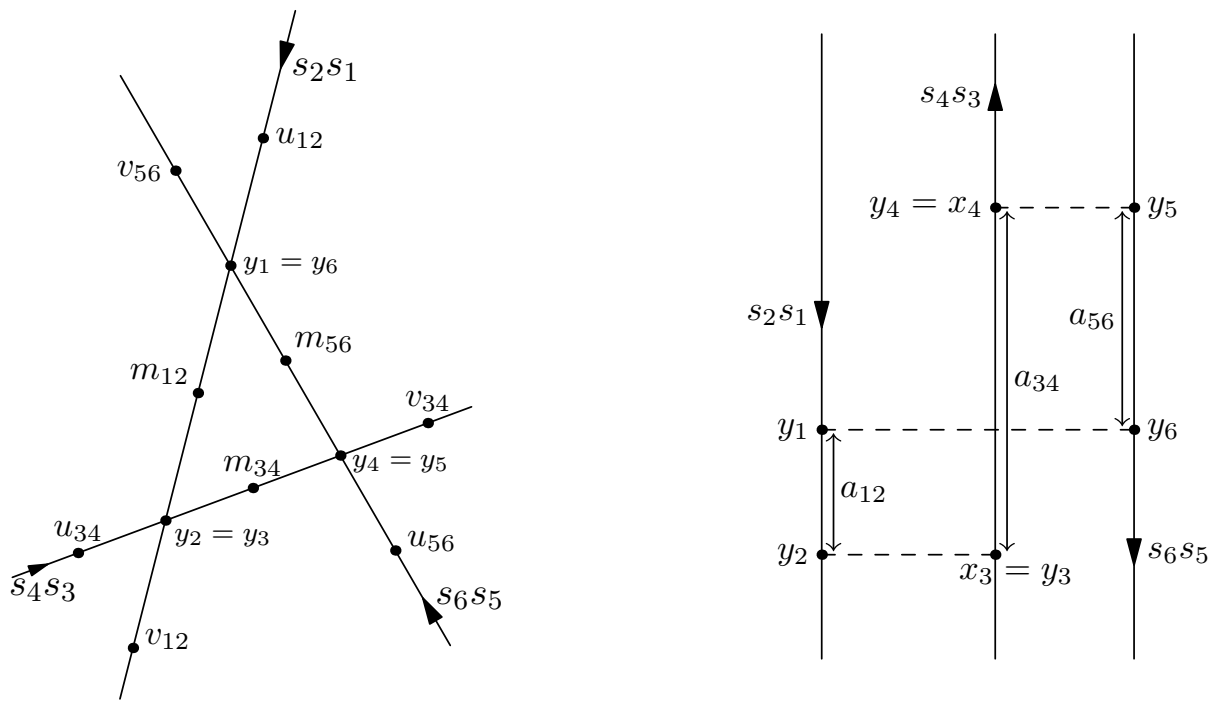

Figure 4. The cases (TRI), to the left, and (PAR), to the right

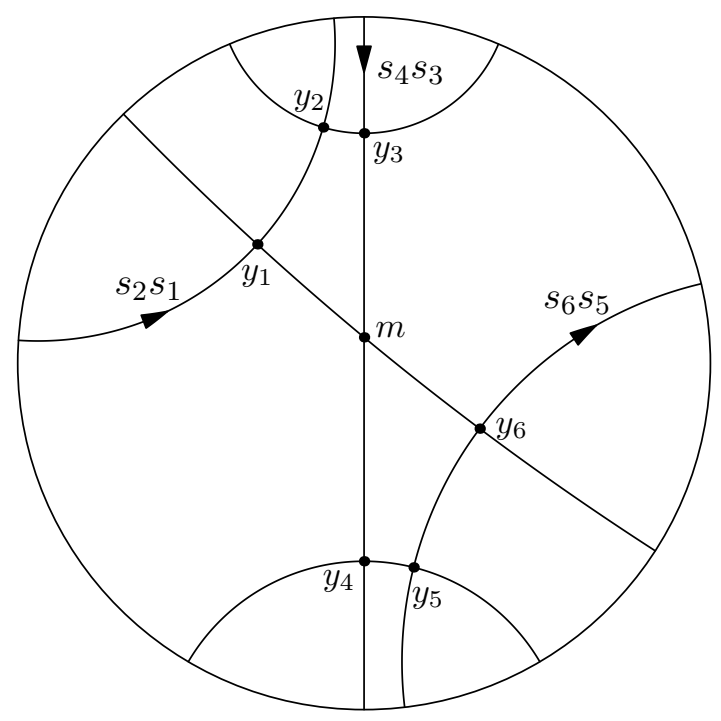

Figure 5. The case (SKH)

functions to measure this size. If $z=\left(x_{1}, \ldots, x_{6}\right) \in \mathrm{Sex}$, as in Section 2.4 let $a_{i, i+1}=d\left(x_{i}, x_{i+1}\right)$ for all $i$ with cyclic notation, and put

$$
F(z)=\prod_{i=1}^{6} \cosh \left(a_{i, i+1}\right), \quad A(z)=a_{12}+a_{34}+a_{56} \quad \text { and } \quad B(z)=a_{23}+a_{45}+a_{61} .
$$

3.1. The space $X_{0}$ is contractible. An elementary computation shows that the differentiable map $\left(\mathbb{H}^{2}\right)^{6} \rightarrow \mathrm{PSL}_{2}(\mathbb{R})$ defined by $\left(x_{1}, \ldots, x_{6}\right) \mapsto s_{x_{6}} \cdots s_{x_{1}}$ has surjective differential at every point such that $x_{i} \neq x_{j}$ for some $i, j$. Thus, the subspace Sex* of non-singular sextuple configurations is a smooth submanifold of $\left(\mathbb{H}^{2}\right)^{6}$, and the map $F: \mathrm{Sex}^{*} \rightarrow \mathbb{R}$ is differentiable. 
Lemma 3.1. The map $F$ has no critical points on $\mathrm{Sex}_{0}^{*}$.

Proof. By contradiction, suppose $z=\left(x_{1}, \ldots, x_{6}\right) \in \operatorname{Sex}_{0}^{*}$ is a critical point for $F$.

If $z$ is an aligned configuration, we can push the points together by rescaling simultaneously $a_{i, i+1}$ for all $i$ by the same factor, thus decreasing $F$ at first order, a contradiction.

Suppose now that $x_{i} \neq x_{i+1}$ for all $i$. Consider the line $D_{12}$, oriented from $x_{1}$ to $x_{2}$ and consider a flow $\phi_{12}^{t}$ on a neighbourhood of $z$ in Sex replacing $x_{1}$ and $x_{2}$ by their image by the translation of length $t$ along $D_{12}$. Let $x_{6}^{\prime}$ and $x_{3}^{\prime}$ be the orthogonal projections of $x_{6}$ and $x_{3}$ on $D_{12}$. By the hyperbolic Pythagorean theorem, we have $\cosh \left(a_{61}\right)=\cosh \left(d\left(x_{6}, x_{6}^{\prime}\right)\right) \cosh \left(d\left(x_{6}^{\prime}, x_{1}\right)\right)$ and $\cosh \left(a_{23}\right)=\cosh \left(d\left(x_{3}, x_{3}^{\prime}\right)\right) \cosh \left(d\left(x_{3}^{\prime}, x_{2}\right)\right)$, hence

$$
F\left(\phi_{12}^{t}(z)\right)=C \cosh \left(d\left(x_{6}^{\prime}, x_{1}\right) \pm t\right) \cosh \left(d\left(x_{2}, x_{3}^{\prime}\right) \pm t\right),
$$

where $C=\cosh \left(a_{12}\right) \cosh \left(a_{34}\right) \cosh \left(a_{45}\right) \cosh \left(a_{56}\right) \cosh \left(d\left(x_{6}, x_{6}^{\prime}\right) \cosh \left(x_{3}, x_{3}^{\prime}\right)\right.$ does not depend on $t$, and where the signs before $t$ depend on the order of the points $x_{1}, x_{2}, x_{6}^{\prime}$ and $x_{3}^{\prime}$ on the line $D_{12}$. In either case, by deriving the above expression, we check that the criticality of $F$ at $z$ implies that the midpoints of the segments $\left[x_{1}, x_{2}\right]$ and $\left[x_{3}^{\prime}, x_{6}^{\prime}\right]$ coincide; similar conclusions hold if we cyclically permute the $x_{i}$ 's.

Put $x=d\left(x_{1}, y_{1}\right), y=d\left(x_{3}, y_{3}\right)$ and $z=d\left(x_{5}, y_{5}\right)$. Note that we also have $x=d\left(x_{2}, y_{2}\right)$, $y=d\left(x_{4}, y_{4}\right)$ and $z=d\left(x_{6}, y_{6}\right)$. So the midpoint of $\left[x_{1}, x_{2}\right]$ is at distance $x$ from $m_{12}$, the midpoint of $\left[y_{1}, y_{2}\right]$.

If we are in case (TRI) or (SKH), the projections of $y_{3}$ and $y_{6}$ on $D_{12}$ are $y_{2}$ and $y_{1}$. Since orthogonal projections decrease distances, the midpoint of $\left[x_{3}^{\prime}, x_{6}^{\prime}\right]$ is at distance less than $\frac{y+z}{2}$ of $m_{12}$, with equality if and only if $y=z=0$. Thus we have the inequality $2 x \leq y+z$, and its three cyclic companions. It follows that $x=y=z=0$. In case (TRI) this contradicts that $x_{2} \neq x_{3}$. In case $(\mathrm{SKH})$ we can shorten $a_{34}$ while fixing $a_{12}$ and $a_{56}$, by classical hyperbolic formulas (see eg [2], page 454) this shortens $a_{23}, a_{45}$ and $a_{61}$, contradicting that $z$ is a critical point of $F$.

If we are in case (PAR), we again contradict that $z$ is a critical point of $F$ by pushing simultaneously all the points $x_{i}$ towards the common point at infinity of $D_{12}, D_{34}$ and $D_{56}$ : this leaves $a_{12}, a_{34}$ and $a_{56}$ invariant while decreasing the other three distances.

Finally, suppose, say, that $x_{1}=x_{2}$. Then $x_{3}, \ldots, x_{6}$ lie in a same line $D$, well-defined since the configuration is not aligned. Let $x$ be the orthogonal projection of $x_{1}$ on $D$. Then we may push simultaneously the points $x_{3}, \ldots, x_{6}$ towards $x$ along $D$, reducing all the distances $a_{i, i+1}$ at the first order, thus $z$ cannot be a critical point of $F$.

Every element of $\mathrm{Sex}^{*}$ has a closed $\mathrm{PSL}_{2}(\mathbb{R})$-orbit; it follows that $X^{*}$ is smooth: the only singularity of $X$ is the singular configuration. It is noteworthy that although the aligned, nonsingular configurations are smooth points of $X^{*}$, their images in $X_{0}(\Gamma)$ are non-smooth points of the character variety of the genus two surface group.

Now, the map $F: \operatorname{Sex}_{0}^{*} \rightarrow(1,+\infty)$ is $\operatorname{PSL}_{2}(\mathbb{R})$-invariant, hence it descends to a map $f: X_{0}^{*} \rightarrow$ $(1,+\infty)$ which still has surjective differential at every point. Obviously $f$ is onto; it is also proper. Therefore, $X_{0}^{*}$ is diffeomorphic to $(1,+\infty) \times f^{-1}(\{r\})$ for any $r>1$, and $X_{0}$ is homeomorphic to a cone over $f^{-1}(\{r\})$. This proves that $X_{0}$ is contractible, and homeomorphic to a neighbourhood of the singular configuration. This neighbourhood will be described precisely in Section 4 .

\subsection{An effective method.}

3.2.1. The set $U$. In this section we consider the subset $U$ of non-pinched isometry classes of sextuple configurations. Before proving Theorem 1.1, let us prove the following statement, which will be needed in Section 5 . 
Observation 3.2. The set $U$ is connected, is dense and has full measure in $X_{0}$.

Proof. Consider the following set $P=\left\{\left(x_{1}, \ldots, x_{6}\right) \in \operatorname{Sex} \mid x_{1}=x_{2}, x_{3}=x_{4}, x_{5}=x_{6}, x_{2} \neq\right.$ $\left.x_{3}\right\}$ of pinched, non-singular configurations. It is a submanifold of dimension 6 of $\mathrm{Sex}^{*}$, and it descends to a submanifold of dimension 3, hence of codimension 3 , of $X_{0}$. Now $U$ is the complement of the orbit of this codimension 3 submanifold, under the countable group $\operatorname{Mod}\left(S_{o}\right)$; the statement of the observation follows.

Now we turn to the proof of Theorem 1.1, restated as follows.

Theorem 3.3. For all $[z] \in U$, the adherence $\overline{\operatorname{Mod}\left(S_{o}\right) \cdot[z]}$ contains the isometry class of singular configurations.

3.2.2. The operations. To prove this statement we show how to construct an effective "geometric algorithm" (we will comment later on this terminology) which reduces the size, measured by the functions $A$ and $B$ introduced above, of sextuple configurations. Suppose $z=\left(x_{1}, \ldots, x_{6}\right)$ is a non-aligned sextuple satisfying $x_{i} \neq x_{i+1}$ for $i=1,3,5$. Consider the following operations on $z$, depending on the trichotomy of Lemma 2.3

(Rot) Make a cyclic permutation, to obtain a sextuple $z^{\prime}$ with $A\left(z^{\prime}\right)=B(z)$ and $B\left(z^{\prime}\right)=A(z)$.

(Tri) If we are in case (TRI), apply (Rot) an even number of times, so that $a_{12} \geq a_{34}$ and $a_{12} \geq a_{56}$. Then perform the leapfrog moves $L_{1}$ to a power minimizing the distance $d\left(x_{1}, y_{1}\right)$; apply similarly the moves $L_{3}$ and $L_{5}$. Then, if this further decreases $B$, apply again $L_{1}$ or its inverse, once. Then apply (Rot).

(Par) If we are in case (PAR), apply leapfrog moves $L_{3}$ to push $x_{3}$ and $x_{4}$ towards the common point at $\partial \mathbb{H}^{2}$ of $D_{12}, D_{34}$ and $D_{56}$, until the distances along horocycles, from $x_{3}$ or $x_{4}$, to $D_{12}$ and $D_{56}$, become less than $\frac{A(z)}{12}$. Then consider the points $y_{1}, y_{2}, y_{5}$ and $y_{6}$ as in Figure 4, and apply powers of $L_{1}$ and $L_{5}$ to minimize the distances $d\left(x_{1}, y_{1}\right)$ and $d\left(x_{5}, y_{5}\right)$. Then apply (Rot).

(Skh0) If we are in case (SKH), first apply (Rot) an even number of times so that $a_{34}>a_{12}+a_{56}$, as in Figure 5. Apply powers of $L_{1}$ and $L_{5}$ to minimize $d\left(x_{1}, y_{1}\right)$ and $d\left(x_{5}, y_{5}\right)$. Apply a power of $L_{3}$ to put $x_{3}$ in the segment $\left[y_{3}, m\right]$ or $x_{4}$ in the segment $\left[m, y_{4}\right]$.

(Skh1) If in case (SKH), apply (Skh0), and then apply the "half-twist move" $\left(L_{1} L_{2} L_{1}\right)^{2}$.

The effect of these operations is precised in the following lemmas.

Lemma 3.4. Suppose $z$ is in case (TRI) or (PAR), and let $z^{\prime}$ be the sextuple resulting from applying the relevant operation, (Tri) or (Par), to $z$. Then $\frac{24}{23} A\left(z^{\prime}\right) \leq B\left(z^{\prime}\right)=A(z)$.

In the next lemmas, we write $b_{i, i+1}=d\left(y_{i}, y_{i+1}\right)$ for $i=2,4,6$, in the case (SKH), so that the right-angled skew hexagon has edge lengths $a_{i, i+1}$ with $i=1,3,5$ and $b_{i, i+1}$ with $i=2,4,6$.

Lemma 3.5. Suppose $z$ is in case (SKH), and let $z^{\prime}$ be the sextuple resulting from applying the operation (Skh1) to $z$. Then $A\left(z^{\prime}\right)<A(z)$. More precisely, if $a_{34}>a_{12}+a_{56}$, then the operation (Skh1) leaves $a_{12}$ and $a_{56}$ invariant and decreases $\cosh \left(a_{34}\right)$ by a quantity larger than $2 \frac{\sinh ^{2}\left(\min \left(a_{12}, a_{56}\right)\right)}{\cosh ^{2}\left(a_{34}\right)}$. Moreover, provided $A(z)$ is small enough, we also have $B\left(z^{\prime}\right) \leq B(z)+$ $4 A(z)-\min \left(1, b_{23}, b_{45}\right)$.

The operations described above do not suffice yet to prove Theorem 1.1, the next lemma introduces an additional operation.

Lemma 3.6 (Operation (Skh2)). Provided $A(z)$ and $\min _{i=2,4,6}\left(b_{i, i+1}\right)$ are small enough, after applying (Skh0) the isometry $s_{x_{3}^{\prime}} s_{x_{2}^{\prime}} s_{x_{1}^{\prime}}$ is a rotation of angle close (but not equal) to $\pi$. Then there exists $N \geq 0$ such that $\left(s_{x_{3}^{\prime}} s_{x_{2}^{\prime}} s_{x_{1}^{\prime}}\right)^{N}$ is a rotation of angle close to $\pm \frac{\pi}{2}$ and such that the 
move $\left(L_{1} L_{2} L_{1}\right)^{2 N}$ results in decreasing $A$, and such that, if the resulting configuration $z^{\prime}$ is still in case (SKH), then after applying again (Skh0) we have $B\left(z^{\prime}\right) \leq B(z)-1$.

3.2.3. Proof of Theorem 1.1. Let $z=\left(x_{1}, \ldots, x_{6}\right) \in$ Sex such that $[z] \in U$, and let $\varepsilon>0$, small enough to apply Lemmas 3.5 and 3.6. We want to prove that there exists $z^{\prime} \in B_{6}\left(S^{2}\right) \cdot z$ such that $A\left(z^{\prime}\right)+B\left(z^{\prime}\right) \leq 2 \varepsilon$.

Let us suppose that the configuration is not aligned; we postpone the aligned case to the end of the proof. The case in which $x_{i}=x_{i+1}$ for some $i$ is quite straightforward and we will deal with it later. Thus, let us suppose now that our configuration $z$, as well as all the configurations we deal with in the following process, satisfy the condition $x_{i} \neq x_{i+1}$ for all $i$.

As a first step, let us apply the operations (Tri) or (Par) or (Skh1), depending on whether $z$ is in case (TRI), (PAR) or (SKH) of Lemma 2.3, and iterate this procedure, until $A \leq \varepsilon$. This first process stops in finite time. Indeed, in cases (TRI) and (PAR), $A$ drops by a factor of at least $\frac{24}{23}$. The worst thing that could happen is to encounter only the case (SKH) after some iteration. Suppose it is the case. By Lemma 3.5, at each iteration, the three quantities $a_{12}, a_{34}$ and $a_{56}$ all decrease, hence the biggest of them stays smaller than the starting quantity $A(z)$. Hence, by Lemma 3.5, at each iteration the biggest of $\cosh \left(a_{12}\right), \cosh \left(a_{34}\right)$ and $\cosh \left(a_{56}\right)$ decreases, by an additive amount depending only on the smallest. Hence, should the process not stop in finite time, the smallest of $a_{12}, a_{34}$ and $a_{56}$ would have to converge to 0. But each iteration changes only the biggest of $a_{12}, a_{34}$ and $a_{56}$. Hence, these three quantities converge to 0 , and this first stage of iterations does stop in finite time.

Now we have $A\left(z^{\prime}\right) \leq \varepsilon$. Of course, under this condition, if we encounter again the case (TRI) or (PAR) then we are done, by Lemma 3.4. Suppose we do not. Proceed as in the first step until we have $\min \left(b_{23}, b_{45}, b_{61}\right) \leq \varepsilon$ in case $(\mathrm{SKH})$. This will happen in finite time, by the second assertion of Lemma 3.5. Note that the operation (Skh0) leaves $A$ invariant, and after this operation we have $B \leq b_{23}+b_{45}+b_{61}+2 A$. So as a last step, we proceed by iterating either the operation (Skh1), or ((Skh2) followed by (Skh0)), depending on which decreases $B$ the most. The conclusions of Lemmas 3.5 and 3.6 now imply that $B$ converges to 0 , hence is lower than $\varepsilon$ after finitely many iterations.

Let us deal now with the case when $x_{i}=x_{i+1}$ for some $i$. Up to applying (Rot), suppose that $x_{5}=x_{6}$. Now $s_{x_{4}} s_{x_{4}} s_{x_{2}} s_{x_{1}}=1$, so $x_{1}, x_{2}, x_{3}$ and $x_{4}$ have to lie on a same line. Since $[z] \in U$, these four points need to be pairwise distinct, otherwise we could easily produce leapfrog moves leading to a configuration where $x_{i}=x_{i+1}$ for $i=1,3,5$. Denote by $\Delta$ be the line containing $x_{1}, \ldots, x_{4}$ and orient this line. Up to applying powers of $L_{3}$ we may suppose that $x_{1}$ is to the left of $x_{2}, x_{3}$ and $x_{4}$ on $\Delta$; this implies that $x_{3}$ is at the right side of $x_{1}, x_{2}, x_{4}$. Now put $\delta_{1}=d\left(x_{1}, x_{2}\right)$ and $\delta_{2}=d\left(x_{1}, x_{4}\right)$. If $\delta_{2}>\delta_{1}$ apply the move $L_{3}$, this changes $\left(\delta_{1}, \delta_{2}\right)$ into $\left(\delta_{1}, \delta_{2}-\delta_{1}\right)$. If $\delta_{1}<\delta_{2}$ apply the move $L_{2}^{-1}$; this changes $\left(\delta_{1}, \delta_{2}\right)$ into $\left(\delta_{1}-\delta_{2}, \delta_{2}\right)$. We recognise Euclid's algorithm. It follows from the condition $[z] \in U$ that $\delta_{1}$ and $\delta_{2}$ have an irrational ratio, so this process pushes the points $x_{1}, x_{2}, x_{3}$ and $x_{4}$ close together. By applying then powers of $L_{1}$ and $L_{3}$ we may now push these four points as close as we want to the projection of $x_{5}=x_{6}$ on $\Delta$. If $x_{5}=x_{6} \in \Delta$ then we are done. Otherwise, according to the construction of Section 2.2. the isometry $s_{x_{6}} s_{x_{1}} s_{x_{2}}$ is a rotation, of center as close as we want from $x_{5}$, and of angle close (but distinct) to $\pi$, hence it has an $N$-th power with angle close to $\frac{\pi}{2}$. Then apply the iterated half-twist, $\left(L_{6} L_{1} L_{6}\right)^{2 N}$. This results in a configuration with $A$ as small as we want (hence $A \leq \varepsilon)$, furthermore in this new configuration the lines $\left(x_{1}, x_{2}\right)$ and $\left(x_{3}, x_{4}\right)$ now cross each other, hence this configuration is in case (TRI). Thus, it remains to apply the operation (Tri) in order to get $A \leq \varepsilon$ and $B \leq \varepsilon$. 
The case of aligned configurations, finally, can be treated by mixing the strategy of case (PAR) and that of the degenerate case above, depending on whether $x_{i}=x_{i+1}$ for some $i$.

\subsubsection{Proofs of the lemmas.}

Proof of Lemma 3.4. The leapfrog moves $L_{1}, L_{3}$ and $L_{5}$ obviously do not change the distances $a_{12}, a_{34}$ and $a_{56}$ summing up to $A(z)$. We need to prove that the moves as in Operations (Tri) and (Par), except the last rotation, lead to a configuration $z^{\prime}$ with $B\left(z^{\prime}\right)<\frac{23}{24} A(z)$. Here we use the notation $z^{\prime}$ even though we may not have reached yet the configuration as in the statement of the lemma; the notation $z^{\prime}$ is subject to change in the course of the proof, accordingly to the appropriate moves. We make this abuse of notation here, and in the two subsequent proofs.

Suppose first that $z$ is in the case (TRI). After applying the leapfrog moves minimizing the distances $d\left(x_{i}^{\prime}, y_{i}\right)$, we have $x_{i}^{\prime} \in\left[u_{i, i+1}, m_{i, i+1}\right]$ for $i=1,3,5$ and $x_{i}^{\prime} \in\left[m_{i-1, i}, v_{i-1, i}\right]$ for $i=2,4,6$, where the points $m_{i, i+1}, u_{i, i+1}$ and $v_{i, i+1}, i=1,3,5$ are as in Figure 4 . Thus,

$$
\begin{array}{lll}
d\left(x_{1}^{\prime}, y_{1}\right) \leq \frac{a_{12}}{2}, & d\left(x_{2}^{\prime}, y_{2}\right) \leq \frac{a_{12}}{2}, & d\left(x_{3}^{\prime}, y_{3}\right) \leq \frac{a_{34}}{2} \\
d\left(x_{4}^{\prime}, y_{4}\right) \leq \frac{a_{34}}{2}, & d\left(x_{5}^{\prime}, y_{5}\right) \leq \frac{a_{56}}{2}, & d\left(x_{6}^{\prime}, y_{6}\right) \leq \frac{a_{56}}{2} .
\end{array}
$$

As $y_{1}=y_{6}, y_{2}=y_{3}$ and $y_{4}=y_{5}$ this already gives $B\left(z^{\prime}\right) \leq A(z)$, by triangular inequalities.

If $B\left(z^{\prime}\right) \geq \frac{23}{24} A(z)$, then the distances of each $x_{i}^{\prime}$ to the closest end of its segment sum up to less than $\frac{A(z)}{24}$. Suppose (without loss of generality) that $a_{56} \leq a_{34} \leq a_{12}$, so that $a_{56} \leq \frac{A(z)}{3}$. The symmetry around $y_{2}$ and the CAT(0) inequality (and the intercept theorem) imply $d\left(v_{12}, u_{34}\right)=$ $d\left(m_{12}, m_{34}\right) \leq \frac{a_{56}}{2}$. If $d\left(x_{2}^{\prime}, m_{12}\right)+d\left(x_{3}^{\prime}, m_{34}\right) \leq \frac{A(z)}{24}$, or $d\left(x_{2}^{\prime}, v_{12}\right)+d\left(x_{3}^{\prime}, u_{34}\right) \leq \frac{A(z)}{24}$, then by triangle inequalities we get $B\left(z^{\prime}\right) \leq \frac{21}{24} A(z)$, a contradiction. Thus $d\left(x_{2}^{\prime}, v_{12}\right)+d\left(x_{3}^{\prime}, m_{34}\right) \leq \frac{A(z)}{24}$ or $d\left(x_{2}^{\prime}, m_{12}\right)+d\left(x_{3}^{\prime}, u_{34}\right) \leq \frac{A(z)}{24}$. After an extra leapfrog move $L_{1}^{ \pm 1}$ we have again $d\left(x_{2}^{\prime}, x_{3}^{\prime}\right) \leq$ $\frac{a_{56}}{2}+\frac{A(z)}{24}$, and now we only have $d\left(x_{1}^{\prime}, y_{1}\right) \leq \frac{a_{12}}{2}+\frac{A(z)}{24}$. Triangle inequalities this time give $B\left(z^{\prime}\right) \leq \frac{22}{24} A(z)$; this settles the triangle case.

The parabolic case is much simpler: the inequalities $d\left(y_{2}, x_{3}^{\prime}\right) \leq \frac{A(z)}{12}, d\left(x_{4}^{\prime}, y_{5}\right) \leq \frac{A(z)}{12}$, $d\left(y_{1}, y_{6}\right) \leq \frac{A(z)}{6}, d\left(x_{i}^{\prime}, y_{i}\right) \leq \frac{a_{12}}{2}$ for $i=1,2$ and $d\left(x_{i}^{\prime}, y_{i}\right) \leq \frac{a_{56}}{2}$ for $i=5,6$ directly yield, by triangle inequalities, $B\left(z^{\prime}\right) \leq \frac{5}{6} A(z)$.

Proof of Lemma 3.5. Let $z=\left(x_{1}, \ldots, x_{5}\right) \in$ Sex be in the (SKH) configuration. Perform the moves according to the operation (Skh1), except the last "half-twist". Without loss of generality, suppose that now $x_{3}^{\prime} \in\left[y_{3}, m\right]$. The moves we made so far do not change the value of $A$, and clearly, the half-twist then does not change the value of $a_{12}$ or $a_{56}$. In order to prove the first statement of Lemma 3.5 we study the effect of this half-twist on $a_{34}$.

This half-twist amounts to replace $x_{1}^{\prime}, x_{2}^{\prime}$ and $x_{3}^{\prime}$ by their image by the isometry $s_{x_{3}^{\prime}} s_{x_{2}^{\prime}} s_{x_{1}^{\prime}}$. Recall from Section 2.2 that this isometry is the product $r_{\ell_{2}} r_{\ell_{1}}$ of the reflections by the lines $\ell_{1}$ and $\ell_{2}$, as constructed in Figure6. Construct, similarly, the lines $\ell_{3}$ and $\ell_{4}$ such that $s_{x_{6}^{\prime}} s_{x_{5}^{\prime}} s_{x_{4}^{\prime}}=$ $r_{\ell_{3}} r_{\ell_{4}}$. As $z \in$ Sex, we have $r_{\ell_{1}} r_{\ell_{2}}=r_{\ell_{3}} r_{\ell_{4}}$. In particular these four lines either intersect in $\mathbb{H}^{2}$, or in $\partial \mathbb{H}^{2}$, or are all perpendicular to a common line, depending on whether $s_{x_{3}^{\prime}} s_{x_{2}^{\prime}} s_{x_{1}^{\prime}}$ is elliptic, parabolic or hyperbolic: this is what we observe in Figure 6, but our reasoning will not depend on this trichotomy.

We want to compare $a_{34}$ with the new distance, $d\left(r_{\ell_{2}} r_{\ell_{1}} \cdot x_{3}^{\prime}, x_{4}^{\prime}\right)$. This last distance is equal to $d\left(x_{3}^{\prime}, r_{\ell_{3}} r_{\ell_{4}} \cdot x_{4}^{\prime}\right)=d\left(x_{3}^{\prime}, r_{\ell_{3}} x_{4}^{\prime}\right)$. Let $p_{3} \in \mathbb{H}^{2}$ and $\theta_{3} \in \mathbb{R}$ be as in Figure 6, the intersection point of $\ell_{3}$ and $D_{34}$ and their angle. Set $u=d\left(x_{3}^{\prime}, p_{3}\right)$ and $v=d\left(p_{3}, x_{4}^{\prime}\right)$. Then $\cosh \left(a_{34}\right)=\cosh u \cosh v+$ 


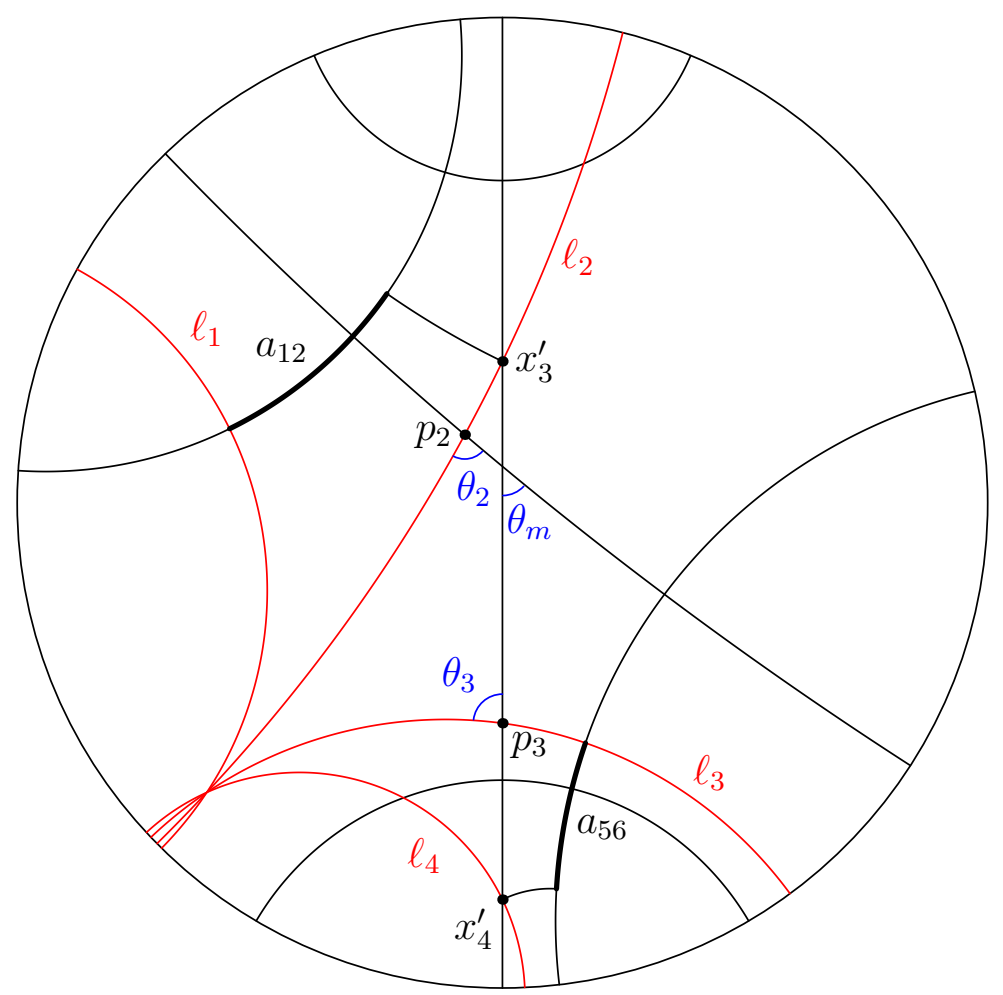

FiguRE 6. The operation (Skh1)

$\sinh u \sinh v$, whereas $\cosh \left(d\left(x_{3}^{\prime}, r_{\ell_{3}} x_{4}^{\prime}\right)\right)=\cosh u \cosh v+\cos \left(2 \theta_{3}\right) \sinh u \sinh v$, hence the decrement in $\cosh \left(a_{34}\right)$ is equal to $2 \sin ^{2} \theta_{3} \sinh u \sinh v$. We claim that $u \geq \min \left(a_{12}, a_{56}\right), v \geq a_{56}$, and $\sin \theta_{3} \geq \frac{1}{\cosh \left(a_{34}\right)}$; these claims imply the first part of Lemma 3.5 .

The inequality $v \geq a_{56}$ is obvious. Also, we have $d\left(p_{3}, y_{4}\right) \leq a_{34}$. Thus, the lines $\ell_{3}, D_{34}$ and $\left(y_{4}, y_{5}\right)$ form the same picture as in Figure 7 , where the angle and the distance involved are $\theta_{3}$ and $d\left(p_{3}, y_{4}\right)$. Indeed, $\ell_{3}$ and $\left(y_{4}, y_{5}\right)$ being both perpendicular to the same line $D_{56}$, do not cross each other. The claimed inequality follows. The remaining inequality, $u=d\left(x_{3}, p_{3}\right) \geq \min \left(a_{12}, a_{56}\right)$,

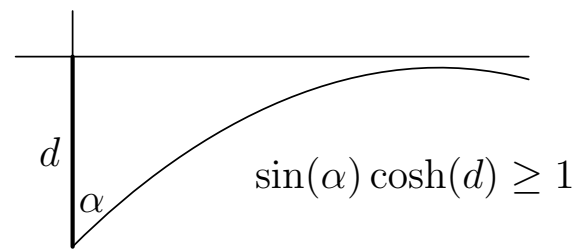

Figure 7. A classical inequality

is obtained by a variation argument. If $x_{3}=y_{3}$ then $p_{3}=m$, so $u \geq a_{12}$. Starting there, as $x_{3}$ moves down, $x_{4}$ moves down at the same speed, and $v$ decreases until it reaches a minimum when $y_{4}$ is the middle of the segment $\left[p_{3}, x_{4}\right]$. It then increases in a symmetric way as $x_{3}$ moves further down, until it reaches its initial value as $p_{3}=y_{4}$. Along the way we always have $u \geq a_{12}$. 
If, in this process, $x_{3}^{\prime}$ has already reached $m$, then we are done. Otherwise, after $p_{3}=y_{4}$ we have $d\left(x_{3}, p_{3}\right) \geq d\left(m, y_{4}\right) \geq a_{56}$. This proves the first part of Lemma 3.5.

Before applying (Skh0) we had $B(z) \geq b_{23}+b_{45}+b_{61}$. Indeed, $y_{2}$ and $y_{3}$ realize the smallest distance between a point in $D_{12}$ and a point in $D_{34}$, and so on. Let us write $\left(x_{1}, \ldots, x_{6}\right)$ the points after applying $(\mathrm{Skh} 0)$, and $\left(x_{1}^{\prime}, \ldots, x_{6}^{\prime}\right)$ the points after applying $\left(L_{1} L_{2} L_{1}\right)^{2}$. Concerning $B$, this last move does not affect $d\left(x_{2}, x_{3}\right)$ or $d\left(x_{4}, x_{5}\right)$; it changes only $d\left(x_{6}, x_{1}\right)$, by applying $r_{\ell_{2}} r_{\ell_{1}}$ to $x_{1}$. We have $d\left(x_{6}, y_{6}\right) \leq \frac{a_{56}}{2}$, and $d\left(r_{\ell_{1}} x_{1}, y_{2}\right) \leq 4 a_{12}$. So $d\left(x_{6}^{\prime}, x_{1}^{\prime}\right) \leq \frac{a_{56}}{2}+4 a_{12}+d\left(r_{\ell_{2}} y_{2}, y_{6}\right)$. Also, $d\left(x_{2}^{\prime}, x_{3}^{\prime}\right) \leq b_{23}+\frac{a_{12}}{2}+a_{34}$ and $d\left(x_{4}^{\prime}, x_{5}^{\prime}\right) \leq b_{45}+a_{34}+\frac{a_{56}}{2}$, so $B\left(z^{\prime}\right) \leq d\left(r_{\ell_{2}} y_{2}, y_{6}\right)+4 A(z)+b_{23}+b_{45}$. Now, we obviously have $d\left(y_{2}, p_{2}\right) \geq b_{23}, d\left(p_{2}, y_{6}\right) \geq b_{45}$ and $\frac{\pi}{2} \geq \theta_{2} \geq \theta_{m}$, with the notation of Figure 6, and $\theta_{m}$ can be forced to be as close to $\frac{\pi}{2}$ as we want provided $A(z)$ is small; this is made precise by Figure 7 . For $i=2,6$ let $q_{i} \in\left[p_{2}, y_{i}\right]$ the point at distance $\delta=\min \left(1, b_{23}, b_{45}\right)$ of $p_{2}$. Provided $\pi-2 \theta_{2}$ is smaller than the angle of an equilateral triangle of side 1 in $\mathbb{H}^{2}$, we have (eg, by CAT (0) inequality) $d\left(r_{\ell_{2}} q_{2}, q_{6}\right) \leq \delta$, hence $d\left(r_{\ell_{2}} y_{2}, y_{6}\right) \leq b_{61}-\delta$ by triangle inequalities; thus finally $B\left(z^{\prime}\right) \leq b_{23}+b_{45}+b_{61}+4 A(z)-\min \left(1, b_{23}, b_{45}\right)$.

Proof of Lemma 3.6. If $A(z)$ is small, and, say, $b_{23}$ is small, then after doing (Skh0), as of the construction of Section 2.2, $s_{x_{3}} s_{x_{2}} s_{x_{1}}$ is a rotation of angle close to $\pi$, with center very close to the segment $\left[x_{3}, x_{4}\right]$. For some $N$, its $N$ th power has angle close to $\frac{\pi}{2}$ and $d\left(\left(x_{x_{3}} s_{x_{2}} s_{x_{1}}\right) x_{3}, x_{4}\right) \leq$ $d\left(x_{3}, x_{4}\right)$. Thus the operation (Skh2) does not increase $A$. Also, the lines $\left(x_{1}^{\prime}, x_{2}^{\prime}\right)$ and $\left(x_{5}^{\prime}, x_{6}^{\prime}\right)$ now cross each other (hence we end in case (TRI)), unless the distance $d\left(y_{2}, y_{6}\right)$ was very big. In that case, the distance between $D_{12}$ and $D_{56}$ decreases significantly by the operation (Skh2), and we see easily that $B\left(z^{\prime}\right) \leq B(z)-1$ after doing (Skh0) once again; this inequality is actually extremely far from sharp.

3.3. Side remarks. We end this section with some remarks on which we chose not to expand too much the exposition in this article.

First, as we said in Paragraph 3.2.2, Theorem 1.1 is proved by iterating an explicit geometric procedure, by compass and straightedge construction. We cannot properly speak of an algorithm only because the data of six points in the plane is not a finite information in terms of a finite alphabet: it seems preferable to speak of a "geometric algorithm", or "real number algorithm". In [5] and [6], J. Gilman and B. Maskit gave such an algorithm to decide whether a given nonelementary representation of the free group of rank 2 in $\mathrm{PSL}_{2}(\mathbb{R})$ is discrete. Using this, the "algorithm" given in the paragraph above decides whether a non-elementary representation of the group $\Gamma_{o}$ is discrete. Indeed, it follows from Margulis' lemma, and Theorem 1.1, that a representation in $\operatorname{Hom}^{\prime}\left(\Gamma_{o}, \mathrm{PSL}_{2}(\mathbb{R})\right)$ can be discrete only if it is pinched, in which case we can run the Gilman-Maskit algorithm on the two generators $s_{x_{3}} s_{x_{1}}$ and $s_{x_{5}} s_{x_{1}}$, which generate an index-two (or index-one, accidentally) subgroup of the image of our representation (the case of non-elementary representations of $\Gamma_{o}$ which kill no $c_{i}$ is easier: all of these representations are discrete). In this regard, we can certainly replace $U$ by the set of non-discrete, non-elementary representations in the statement of Theorem 1.1. The following statement, more general and in the spirit of [15] seems reasonable:

Conjecture 3.7. For every non-elementary, non-discrete representation $\rho: \Gamma_{o} \rightarrow \mathrm{PSL}_{2}(\mathbb{R})$, the orbit $B_{6}\left(S^{2}\right) \cdot[\rho]$ is dense in $X_{0}$.

If we replace $\mathrm{PSL}_{2}(\mathbb{R})$ by $\mathrm{PSL}_{2}(\mathbb{C})$, we have a natural identification between the space of nonelementary morphisms of $\Gamma_{o}$ in $\mathrm{PSL}_{2}(\mathbb{C})$ which kill no $c_{i}$, and all the non-elementary morphisms of $\Gamma$ in $\mathrm{PSL}_{2}(\mathbb{C})$ of Stiefel-Whitney class 0; this follows for instance from the arguments of [13], Section 3. These representations of $\Gamma_{o}$ send each generator $c_{i}$ to a rotation of angle $\pi$ around some line in $\mathbb{H}^{3}$. The representations treated in this article correspond to six lines orthogonal 
to a common plane. The representations in $X_{0}^{-}(\Gamma)$ correspond to configurations of six lines in a plane; the remaining real characters of representations in $S O(3)$ correspond to six lines through a common point. The quickest proof of the connectedness of $X_{0}^{-}(\Gamma)$, to our mind, is through this correspondence, by writing, in that setting, the analog of Lemma 2.3, this is all elementary and we leave the details to the reader. We can extend the methods of Theorem 1.1 to these representations in $X_{0}^{-}(\Gamma)$, leading to a much nicer proof of Theorem 1.4 of [13] in the case of Euler class 0; we chose not to elaborate on this point in this article. It seems more interesting, but also quite challenging, to find a subset of representations in $\mathrm{PSL}_{2}(\mathbb{C})$, of positive measure, on which Theorem 1.1 could extend.

\section{Neighbourhood OF THE Singular REPRESENTAtion}

We saw in Theorem 1.1 that the orbit of almost every representation in $X_{0}\left(\Gamma_{o}\right)$ accumulates to the singular representation. The classification of ergodic components of the character varieties therefore reduces to a careful study of a neighbourhood of the singular representation in $X_{0}\left(\Gamma_{o}\right)$. This neighbourhood turns out to have a very rich structure; we devote this section to studying it. This will provide all the material needed to prove the ergodicity statements in Section 5 ,

4.1. A Euclidean model. Suppose that $z^{n}=\left(x_{1}^{n}, \ldots, x_{6}^{n}\right)$ is a sequence of sextuples converging to the singular configuration. Then, up to extraction and renormalization by a scalar, one can suppose that the family $z^{n}$ converges in Gromov-Hausdorff sense to a configuration $\left(p_{1}, \ldots, p_{6}\right)$ in the Euclidean plane $\mathcal{E}$. Consider the set of all limiting configurations up to affine isometry respecting the orientation: the condition $s_{6}^{n} \cdots s_{1}^{n}=1$ implies the same condition for the Euclidean $\pi$-rotations over the $p_{i}^{\prime} s$. This is equivalent to the condition $\sum(-1)^{i} p_{i}=0$. Almost every Euclidean configuration is triangular, and these limits of hyperbolic configurations also have to satisfy an extra condition reminiscent from Remark 2.1. We leave it to the reader as a pleasant exercise in plane Euclidean geometry that this condition is equivalent to the equality between signed areas as appearing in the following definition:

$$
X_{\mathcal{E}}=\left\{\left(p_{1}, \ldots, p_{6}\right) \in \mathcal{E}^{6} / \sum(-1)^{i} p_{i}=0, \operatorname{Area}\left(p_{1}, p_{2}, p_{3}\right)+\operatorname{Area}\left(p_{4}, p_{5}, p_{6}\right)=0\right\} / \operatorname{Isom}^{+}(\mathcal{E}) .
$$

This space is the quotient by a circle action of a quadratic cone inside some vector space $V$. More precisely, consider the space $V=\left\{v=\left(z_{1}, \ldots, z_{6}\right) \in \mathbb{C}^{6} / \sum(-1)^{i} z_{i}=0\right\} / \mathbb{C}$ where $\mathbb{C}$ acts on $\mathbb{C}^{6}$ by diagonal translation, and define on $V$ a Hermitian form $h$ as follows:

$$
\begin{aligned}
h(v, v) & =\operatorname{Area}\left(z_{1}, z_{2}, z_{3}\right)+\operatorname{Area}\left(z_{4}, z_{5}, z_{6}\right) \\
& =\frac{1}{2} \operatorname{det}\left(z_{2}-z_{1}, z_{3}-z_{1}\right)+\frac{1}{2} \operatorname{det}\left(z_{5}-z_{4}, z_{6}-z_{4}\right) \\
& =\frac{1}{2} \sum_{1 \leq i<j \leq 6}(-1)^{i+j+1} \operatorname{det}\left(z_{i}, z_{j}\right)=\frac{1}{2} \sum_{1 \leq i<j \leq 6}(-1)^{i+j} \operatorname{Im}\left(z_{i} \overline{z_{j}}\right) \\
& =\frac{1}{4 i} \sum_{i<j}(-1)^{i+j}\left(z_{i} \overline{z_{j}}-\overline{z_{i}} z_{j}\right) .
\end{aligned}
$$

Also, put $q(v)=h(v, v)$ the underlying real quadratic form and set $C=q^{-1}(0)$. The subset of non-aligned sextuples in $C$ will be denoted by $C^{\times}$. With this notation, $X_{\mathcal{E}}=C / S^{1}$ where $S^{1}$ acts diagonally on $V$. A simple computation shows that $h$ has signature $(2,2)$ on $V$; in particular it is non-degenerate and its imaginary part gives a symplectic form on the real vector space $V$, such that $q$ is a moment map for the diagonal action of $S^{1}$. This implies that $X_{\mathcal{E}}$ has a natural symplectic form, being a symplectic quotient; see [14], Section 5.1 for a reminder. 
This symplectic structure has the property that the Hamiltonian flow of the length function $d\left(z_{1}, z_{2}\right)$ (for instance) is the transformation fixing $z_{3}, z_{4}, z_{5}, z_{6}$ and translating $z_{1}$ and $z_{2}$ along the line joining them. Moreover, the Hamiltonian flow of the function Area $\left(z_{1}, z_{2}, z_{3}\right)$ is given by

$$
\Psi_{123}^{t}\left(z_{1}, \ldots, z_{6}\right)=\left(R_{t} z_{1}, R_{t} z_{2}, R_{t} z_{3}, z_{4}, z_{5}, z_{6}\right)
$$

where $R_{t}$ is the rotation around the point $z_{1}-z_{2}+z_{3}$ (see Section 2.2).

4.2. The Zariski tangent space. From now on in this section, it will be more convenient to replace $\mathrm{PSL}_{2}(\mathbb{R})$ by its isomorphic group $\mathrm{PU}(1,1)=\left\{ \pm\left(\begin{array}{ll}a & b \\ \bar{b} & \bar{a}\end{array}\right), a, b \in \mathbb{C},|a|^{2}-|b|^{2}=1\right\}$ acting by homographies on the unit disc. At a representation $\rho$, the Zariski tangent space to $\operatorname{Hom}\left(\Gamma_{o}, \mathrm{PU}(1,1)\right)$ may be described as the space of paths $\rho_{t}: \gamma \mapsto \exp (t u(\gamma)) \rho(\gamma)$ which, at first order, keep being representations of $\Gamma_{o}$. This condition amounts to the relation $u\left(\gamma_{1} \gamma_{2}\right)=$ $u\left(\gamma_{1}\right)+\operatorname{Ad}_{\rho}\left(\gamma_{1}\right) \cdot u\left(\gamma_{2}\right)$ for all $\gamma_{1}, \gamma_{2}$ where we have set $\operatorname{Ad}_{\rho}(\gamma) \cdot \xi=\rho(\gamma) \xi \rho(\gamma)^{-1}$; the set of such maps $u$ is the space $Z^{1}\left(\Gamma_{o}, \operatorname{Ad}_{\rho}\right)$ of cocycles in group cohomology with coefficients in $\mathrm{pu}(1,1)$ twisted by the adjoint action of $\rho$. Coboundaries, of the form $\gamma \mapsto u_{0}-\operatorname{Ad}_{\rho}(\gamma) u_{0}$, correspond to (actual) deformations of $\rho$ by conjugation, and the Zariski tangent space to $X\left(\Gamma_{o}\right)$ at a class $[\rho]$ is expected to be isomorphic to $H^{1}\left(\Gamma_{o}, \mathrm{Ad}_{\rho}\right)$, see eg [16, 7]. This isomorphism holds at nonelementary representations, by Proposition 5.2 of [10]. In particular, if we denote by the same letter $\rho$ a representation of $\Gamma_{o}$ and the corresponding representation of $\Gamma$, then Proposition 2.1 implies that $H^{1}\left(\Gamma_{o}, \mathrm{Ad}_{\rho}\right) \simeq H^{1}\left(\Gamma, \mathrm{Ad}_{\rho}\right)$ at every non-elementary representation.

At singular representations it may happen however that the Zariski tangent is not isomorphic to this cohomology group. We do not investigate this question here, as we are not concerned with the algebraic structure of $X\left(\Gamma_{o}\right)$.

At the singular representation, this cohomology group has a simpler description as we explain here. Define $\rho_{0}: \Gamma_{o} \rightarrow \mathrm{PU}(1,1)$ by $\rho\left(c_{i}\right)=s_{0}= \pm\left(\begin{array}{cc}i & 0 \\ 0 & -i\end{array}\right)$, the half-turn around 0 , for $i=1, \ldots, 6$. In the natural decomposition $\operatorname{pu}(1,1)=\left\{\left(\begin{array}{cc}i x & z \\ z & -i x\end{array}\right), x \in \mathbb{R}, z \in \mathbb{C}\right\} \simeq \mathbb{R} \oplus \mathbb{C}$, the element $\operatorname{Ad}\left(s_{0}\right)$ acts trivialy on $\mathbb{R}$ and by multiplication by -1 on $\mathbb{C}$, so the cocycle condition implies (with $\gamma_{1}=\gamma_{2}=c_{i}$ for all $i$ ) that every cocycle has a trivial $\mathbb{R}$-part, and (with six terms) that $\sum_{i}(-1)^{i} u\left(c_{i}\right)=0$, whereas a coboundary sends each $c_{i}$ to the same complex number $2 u_{0}$; this gives the natural identification $V \simeq H^{1}\left(\Gamma_{o}, \operatorname{Ad}_{\rho_{0}}\right) \simeq H^{1}\left(\Gamma_{o}, \epsilon\right)$ where by $\epsilon$ we mean $\mathbb{C}$-coefficients twisted by the action $\epsilon\left(c_{i}\right) z=-z$ for $i=1, \ldots, 6$. This is consistent in idea with the beginning of Paragraph 4.1, Indeed, the matrix $\exp \left(\begin{array}{cc}0 & z \\ \bar{z} & 0\end{array}\right) s_{0}= \pm\left(\begin{array}{cc}i \cosh (\rho) & -i \sinh (\rho) e^{i \theta} \\ i \sinh (\rho) e^{-i \theta} & -i \cosh (\rho)\end{array}\right)$, where $z=\rho e^{i \theta}$, acts on the disc by a half-turn around the point $\tanh \left(\frac{\rho}{2}\right) e^{i \theta}$. Thus, if $u$ is an element of $H^{1}\left(\Gamma_{o}, \epsilon\right)$, the associated deformation $\rho_{t}$ maps, at first order, $c_{i}$ to the half-turn around $\frac{t}{2} u\left(c_{i}\right)$.

Now the inclusion map $\Gamma \rightarrow \Gamma_{o}$ yields a map $Z^{1}\left(\Gamma_{o}, \epsilon\right) \rightarrow Z^{1}(\Gamma, \mathbb{C})$ where the $\mathbb{C}$-coefficients are not twisted any more since $\Gamma=\operatorname{ker}(\epsilon)$. By checking on a basis, we will prove that this map induces an isomorphism as follows.

Proposition 4.1. There is a natural isomorphism between $V$ and the cohomology space $H^{1}(\Sigma, \mathbb{C})$ such that the Hermitian form $h$ corresponds to the form $\frac{1}{4 i} v \cdot \bar{w}$ where $\cdot$ denotes the cup product evaluated at the fundamental class.

Proof. Let $\gamma_{1}, \ldots, \gamma_{6}$ be as in Figure 8 and let $\gamma_{i}^{\sharp} \in H^{1}(\Sigma, \mathbb{C})$ be the Poincaré duals of the corresponding cycles. From the signed intersections of the $\gamma_{i}$, we can read $\gamma_{i}^{\sharp} \cdot \gamma_{i+1}^{\sharp}=-1$, $\gamma_{i}^{\sharp} \cdot \gamma_{i-1}^{\sharp}=1$ for all $i$ (with cyclic notation), and $\gamma_{i}^{\sharp} \cdot \gamma_{j}^{\sharp}=0$ if $j \notin\{i-1, i+1\} \bmod 6$. By 
definition $\gamma_{i}^{\sharp}$ is the morphism mapping $\gamma_{j}$ to $\gamma_{j}^{\sharp} \cdot \gamma_{i}^{\sharp}$; it is the image of the cocycle in $Z^{1}\left(\Gamma_{o}, \epsilon\right)$ mapping $c_{i}$ and $c_{i+1}$ to 1 and $c_{j}$ to 0 for $j \neq i, i+1$. As the oriented curves $\gamma_{1}, \gamma_{3}$ and $\gamma_{5}$ (resp. $\gamma_{2}$, $\gamma_{4}$ and $\gamma_{6}$ ) bound a subsurface, we have $\gamma_{1}^{\sharp}+\gamma_{3}^{\sharp}+\gamma_{5}^{\sharp}=0$ and $\gamma_{2}^{\sharp}+\gamma_{4}^{\sharp}+\gamma_{6}^{\sharp}=0$; these identities yield the kernel of the surjective morphism $f_{1}: \mathbb{C}^{6} \rightarrow H^{1}(\Sigma, \mathbb{C})$ defined by $f_{1}\left(\lambda_{1}, \ldots, \lambda_{6}\right)=\sum_{i} \lambda_{i} \gamma_{i}^{\sharp}$. The map $f_{2}: \mathbb{C}^{6} \rightarrow V$ defined by $f_{2}\left(\lambda_{1}, \ldots, \lambda_{6}\right)=\left[\left(\lambda_{1}+\lambda_{2}, \lambda_{2}+\lambda_{3}, \ldots, \lambda_{6}+\lambda_{1}\right)\right]$ obviously
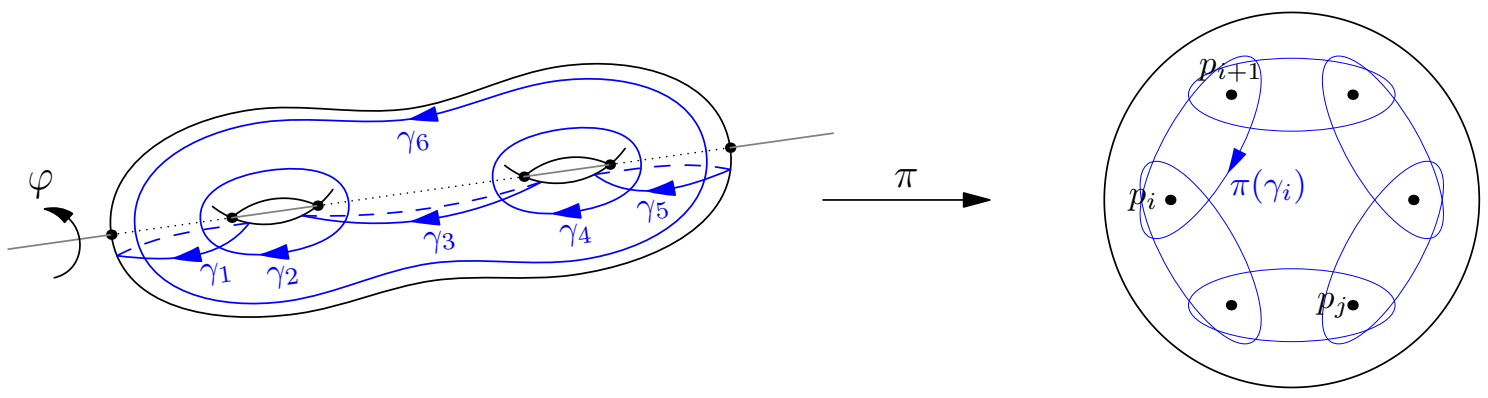

Figure 8. Cycles on the sphere and their lifts

vanishes on $\operatorname{ker} f_{1}$, hence $\operatorname{ker} f_{1}=\operatorname{ker} f_{2}$, so $f_{1}$ and $f_{2}$ induce an isomorphism $V \simeq H^{1}(\Sigma, \mathbb{C})$. Straightforward computation gives, for $\lambda=\left(\lambda_{1}, \ldots, \lambda_{6}\right)$, that $f_{1}(\lambda) \cdot f_{1}(\bar{\lambda})=-2 i \sum_{i} \operatorname{Im} \lambda_{i} \overline{\lambda_{i+1}}$ and $q\left(f_{2}(\lambda)\right)=-\frac{1}{2} \sum_{i} \operatorname{Im} \lambda_{i} \overline{\lambda_{i+1}}$, so the two Hermitian forms coincide.

Remark 4.1. - Viewed as a subgroup of $\operatorname{Aut}\left(\Gamma_{o}\right)$ as we presented it in Section 2.1, our leapfrog group has an obvious action on $V$. However, viewed as $B_{6}\left(S^{2}\right) \simeq \operatorname{Mod}(\Sigma) /[\varphi]$, it acts on $X_{0}\left(\Gamma_{o}\right)$ but not on the Zariski tangent space at $\left[\rho_{0}\right]$, as the hyperelliptic involution acts by -1 ; this is an interesting subtlety.

- Let $\xi \in H^{1}\left(\Gamma_{o}, \operatorname{Ad}_{\rho_{0}}\right)$ represent a tangent vector in $V$. The cup-bracket $[\xi \wedge \xi] \in$ $H^{2}\left(\Gamma_{o}, \operatorname{Ad}_{\rho_{0}}\right)=H^{0}\left(\Gamma_{o}, \operatorname{Ad}_{\rho_{0}}\right)^{*}=H^{0}\left(\Gamma_{o}, \mathbb{R}\right)^{*}=\mathbb{R}$ is an obstruction to $\xi$ being the tangent vector of a deformation of the singular representation. This is a very natural description of the quadratic form $q$ which will not be used in this article.

For the simplicity of the exposition, let us explicit the symplectic form mentioned above. At a point $v \neq 0$ in $C$, the tangent space to $C$ is $\{w \in V \mid \operatorname{Re} h(v, w)=0\}$, so in $X_{\mathcal{E}}$, the tangent space at $[v]=S^{1} v$ is the quotient

$$
T_{[v]} X_{\mathcal{E}}=\{w \in V \mid \operatorname{Re} h(v, w)=0\} / \mathbb{R} i v ;
$$

now if $\left[w_{1}\right],\left[w_{2}\right] \in T_{[v]} X_{\mathcal{E}}$ the formula $\operatorname{Im} h\left(w_{1}, w_{2}\right)$ is well-defined. In terms of the identification above with $H^{1}\left(\Gamma_{o}, \operatorname{Ad}_{\rho_{0}}\right)$, the vector $w_{i}=\left(\lambda_{1}^{i}, \ldots, \lambda_{6}^{i}\right)$ corresponds to the map $\zeta_{i}: \Gamma \rightarrow \operatorname{pu}(1,1)$, $\gamma_{j} \mapsto\left(\begin{array}{cc}0 & \lambda_{j}^{i} \\ \lambda_{j}^{i} & 0\end{array}\right)$. Now the cup-product of $\zeta_{1}$ and $\zeta_{2}$ composed with the Killing form can be computed with the formula $\operatorname{Tr}\left(\begin{array}{cc}0 & z \\ \bar{z} & 0\end{array}\right)\left(\begin{array}{cc}0 & w \\ \bar{w} & 0\end{array}\right)=2 \operatorname{Re} z \bar{w}$, giving $\operatorname{Tr}\left(\zeta_{1} \cdot \zeta_{2}\right)=-8 \operatorname{Im} h\left(w_{1}, w_{2}\right)$.

4.3. The Morse Lemma. In this section, we prove that there is a neighbourhood of $\left[z_{0}\right]$ in $X_{0}$ which is homeomorphic to $X_{\mathcal{E}}$ and diffeomorphic out of the singular configuration. In the identification $V=H^{1}(\Sigma, \mathbb{C})=\operatorname{Hom}\left(H_{1}(\Sigma, \mathbb{R}), \mathbb{C}\right)$, the subspace $C^{\times}$corresponds to surjective maps $u: H_{1}(\Sigma, \mathbb{R}) \rightarrow \mathbb{C}$. We have the following proposition. 
Proposition 4.2. There exist neigbourhoods $U$ of 0 in $C$, and $W$ of $\rho_{0}$ in $\operatorname{Hom}\left(\Gamma_{o}, \mathrm{PU}(1,1)\right)$ which are $S^{1}$-invariant, and an $S^{1}$-equivariant homeomorphism $f: U \rightarrow W$ which

(1) is a diffeomorphism on $U \backslash\{0\}$ to $W \backslash\left\{\rho_{0}\right\}$,

(2) induces a diffeomorphism between $(U \backslash\{0\}) / S^{1}$ and $\left(W \backslash\left\{\rho_{0}\right\}\right) / S^{1}$,

(3) maps bijectively $C^{\times} \cap U$ to $\operatorname{Hom}^{\times}\left(\Gamma_{o}, \mathrm{PU}(1,1)\right) \cap W$.

Proof. In this proof, we denote $s_{0}=\left(\begin{array}{cc}i & 0 \\ 0 & -i\end{array}\right)$ and for any $z_{1}, \ldots, z_{6} \in \mathbb{C}$ we set $\xi_{j}=\left(\begin{array}{cc}0 & z_{j} \\ z_{j} & 0\end{array}\right)$. We will look for a representation $\rho \in \operatorname{Hom}\left(\Gamma_{o}, \mathrm{PU}(1,1)\right)$ such that $\rho\left(c_{j}\right)= \pm \exp \left(\xi_{j}\right) s_{0}$. For that reason, we define in a neighbourhood of 0 a map $F: \mathbb{C}^{6} \rightarrow \mathbb{C}$ and a map $\varphi: \mathbb{C}^{6} \rightarrow \mathbb{R}$ by the formula

$$
\exp \left(\xi_{6}\right) s_{0} \cdots \exp \left(\xi_{1}\right) s_{0}=-\exp \left(\begin{array}{cc}
\frac{i \varphi}{F} & F \\
-i \varphi
\end{array}\right)
$$

We observe that conjugating the equation by the matrix $\left(\begin{array}{cc}e^{i \theta} & 0 \\ 0 & e^{-i \theta}\end{array}\right)$ changes $z_{j}$ to $e^{2 i \theta} z_{j}, F$ to $e^{2 i \theta} F$ and does not change $\varphi$. Hence the map $\varphi$ is $S^{1}$-invariant and the map $F$ is $S^{1}$-equivariant.

We have $-\exp \left(\xi_{6}\right) \cdots s_{0} \exp \left(\xi_{1}\right) s_{0}=\exp \left(\xi_{6}\right) \exp \left(-\xi_{5}\right) \exp \left(\xi_{4}\right) \exp \left(-\xi_{3}\right) \exp \left(\xi_{2}\right) \exp \left(-\xi_{1}\right)$ and its logarithm is $\sum_{i}(-1)^{i} \xi_{i}+\sum_{j>i} \frac{1}{2}\left[(-1)^{j} \xi_{j},(-1)^{i} \xi_{i}\right]$ up to order 2 terms thanks to the BakerCampbell-Hausdorff formula. Hence, the Taylor expansion gives $F\left(z_{1}, \ldots, z_{6}\right)=\sum_{i=1}^{6}(-1)^{i} z_{i}+$ $o(|z|)$ and $\varphi\left(z_{1}, \ldots, z_{6}\right)=-2 q(z)+o\left(|z|^{2}\right)$.

Consider the map $H: \mathbb{C}^{6} \rightarrow \mathbb{C}^{6}$ given by $H\left(z_{1}, \ldots, z_{6}\right)=\left(z_{1}, \ldots, z_{5}, F\left(z_{1}, \ldots, z_{6}\right)\right)$. By the inverse function theorem, this is a local diffeomorphism. We observe that by construction, the map $F$ is $S^{1}$-equivariant, hence the map $H$ and its inverse are also $S^{1}$-equivariant. For small enough $w^{\prime} s$, write $\psi\left(w_{2}, \ldots, w_{5}\right)=\varphi\left(H^{-1}\left(0, w_{2}, w_{3}, w_{4}, w_{5}, 0\right)\right)$. The $S^{1}$-invariant function $\psi$ : $\mathbb{C}^{4} \rightarrow \mathbb{R}$ has a non-degenerate Hessian at 0 and vanish identically if the $w_{i}^{\prime} s$ are real. We conclude by applying the Morse Lemma 4.3. Indeed, if we denote by $\Phi$ the diffeomorphism provided by the lemma, we simply set $f\left(w_{2}, w_{3}, w_{4}, w_{5}\right)=H^{-1}\left(0, x_{2}, x_{3}, x_{4}, x_{5}, 0\right)$ where $\left(x_{2}, x_{3}, x_{4}, x_{5}\right)=$ $\Phi\left(w_{2}, w_{3}, w_{4}, w_{5}\right)$.

The sextuples $\left(z_{1}, \ldots, z_{6}\right) \in \mathbb{R}^{6}$ correspond to linear maps $v: H_{1}(\Sigma, \mathbb{R}) \rightarrow \mathbb{C}$ with values in $\mathbb{R}$. Hence, the $S^{1}$-orbit of real configurations correspond precisely to linear maps of rank 0 or 1 and the diffeomorphism $f$ preserve aligned configurations as expected.

Observe that in the above computation, $q$ appears as a second order obstruction for a cocycle from being realized by deformations of representations; this is yet another language for understanding this quadratic form.

Lemma 4.3 (Equivariant Morse Lemma). Let $\varphi: \mathbb{C}^{n} \rightarrow \mathbb{R}$ be a smooth $S^{1}$-invariant function, vanishing on $\mathbb{R}^{n}$ and such that $\varphi(z)=Q(z)+o\left(|z|^{2}\right)$ for a non-degenerate Hermitian form $Q$. Then there exist $S^{1}$-invariant neighbourhoods $U$ and $V$ of 0 in $\mathbb{C}^{n}$ and an $S^{1}$-equivariant diffeomorphism $\Phi: U \rightarrow V$ such that

- $D_{0} \Phi$ is the identity,

- $\Phi$ preserves $\mathbb{R}^{n}$,

- $\varphi \circ \Phi(z)=Q(z)$ for $z \in U$.

Proof. This is a variation of the standard Morse Lemma with the same proof, using Moser's trick, see [12], Theorem 3.44. It is sufficient to check that the solution provided by the proof has the properties required by the lemma.

We observe that the space $X^{\times}\left(\Gamma_{o}\right)$ as a subspace of $X(\Sigma)$ is endowed with the Atiyah-Bott symplectic structure denoted by $\omega_{A B}$. On the other hand, we explained that $X_{\mathcal{E}}$ is also symplectic 
with a symplectic structure denoted by $\omega$. We do not know whether one can make the local diffeomorphism $f$ symplectic but we will at least need the following weaker statement:

Lemma 4.4. The map $f$ constructed in Proposition 4.2 is a symplectomorphism at first order by which we mean that the following holds:

$$
\left(f^{*} \omega_{A B}\right)_{v}=-8 \omega+o(v) .
$$

Proof. Following Goldman (see [7]), the Atiyah-Bott structure at $[\rho] \in X^{\times}(\Sigma)$ is induced by the cup-product on $H^{1}\left(\Sigma, \operatorname{Ad}_{\rho}\right)$ followed by the trace. The claimed approximation follows from Proposition 4.2 and the computation ending Subsection 4.2 .

4.4. The Grassmannian of Lagrangians. There is yet another description of the space $X_{\mathcal{E}}^{\times}$ of non-aligned Euclidean configurations which will be crucial in the last step of the proof of Theorem 1.2. It uses the Grassmannian of Lagrangians in $H_{1}(\Sigma, \mathbb{R})$ denoted by $\mathcal{L}$. It is a 3 -dimensional manifold and the tangent space at $L \subset H_{1}(\Sigma, \mathbb{R})$ is canonically isomorphic to the space of quadratic forms on $L$. Indeed, the tangent space at $L$ to the Grassmannian of 2-planes is canonically isomorphic to the space $\operatorname{Hom}\left(L, H_{1}(\Sigma, \mathbb{R}) / L\right) \simeq \operatorname{Hom}\left(L, L^{*}\right)$, where we identify $H_{1}(\Sigma, \mathbb{R}) / L$ with $L^{*}$ by the symplectic pairing, and the Lagrangian condition amounts to the symmetry of the corresponding bilinear maps. Dually, the cotangent space of $\mathcal{L}$ at $L$ is isomorphic to the space of quadratic forms on the dual space $L^{*}$. We denote by $T_{+}^{*} \mathcal{L} \subset T^{*} \mathcal{L}$ the set of pairs $(L, \alpha)$ where $\alpha$ is a positive definite quadratic form on $L^{*}$.

Proposition 4.5. There is a diffeomorphism $\Lambda: X_{\mathcal{E}}^{\times} \rightarrow T_{+}^{*} \mathcal{L}$ which is equivariant with respect to the action of $\operatorname{Sp}\left(H_{1}(\Sigma, \mathbb{R})\right)$.

Proof. Recall that an element of $X_{\mathcal{E}}^{\times}$is the $S^{1}$-orbit of a surjective linear map $u: H_{1}(\Sigma, \mathbb{R}) \rightarrow \mathbb{C}$ satisfying $q(u)=0$. Set $L=\operatorname{ker} u$ and show that $q(u)=0$ if and only if $L$ is Lagrangian.

The quantity $q(u)$ is computed from any symplectic basis $a_{1}, b_{1}, a_{2}, b_{2}$ of $H_{1}(\Sigma, \mathbb{R})$ by the formula $q(u)=\frac{1}{4} \operatorname{Im}\left(u\left(a_{1}\right) \overline{u\left(b_{1}\right)}+u\left(a_{2}\right) \overline{u\left(b_{2}\right)}\right)$. If $L$ is Lagrangian, we can ensure that $a_{1}, a_{2}$ form a basis of $L$ and hence $u\left(a_{1}\right)=u\left(a_{2}\right)=0$ and $q(u)=0$. If $L$ is not Lagrangian, it is symplectic and we can form a basis of $L$ with $a_{1}$ and $b_{1}$, which implies that $u\left(a_{2}\right)$ and $u\left(b_{2}\right)$ are linearly independent and hence $q(u) \neq 0$.

Hence, given $u: H_{1}(\Sigma, \mathbb{R}) \rightarrow \mathbb{C}$ with $q(u)=0$ and $L$ its (Lagrangian) kernel, the expression $\alpha(x)=|u(x)|^{2}$ is a positive definite quadratic form on $H_{1}(\Sigma, \mathbb{R}) / L \simeq L^{*}$, hence $\alpha$ belongs to $T_{+}^{*} L$ and does not change if we multiply $u$ by a phase. This construction can be easily reversed and is symplectically invariant hence the map $\Lambda: u \mapsto(L, \alpha)$ has the required properties.

Lemma 4.6. The map $\Lambda$ is a symplectomorphism up to a constant.

Proof. Let $(L, g)$ be any point in $T_{+}^{*} \mathcal{L}$, one can find a symplectic basis such that $L=\operatorname{Span}\left(a_{1}, a_{2}\right)$ and $a_{1}^{*}, a_{2}^{*}$ is an orthonormal basis of $L^{*}$ with respect to $g$. The map $u=\Lambda^{-1}(L, g)$ is given by $u\left(a_{1}\right)=u\left(a_{2}\right)=0, u\left(b_{1}\right)=1, u\left(b_{2}\right)=i$. There is a local coordinate system $\left(p_{i}, q_{i}\right)_{i=1,2,3}$ on $T^{*} \mathcal{L}$ given by setting $L_{p}=\mathbb{R} e_{1} \oplus \mathbb{R} e_{2}$ where $e_{1}=\left(1,0, p_{1}, p_{2}\right), e_{2}=\left(0,1, p_{2}, p_{3}\right)$ and $g_{q}$ has the matrix $\left(\begin{array}{ll}q_{1} & q_{2} \\ q_{2} & q_{3}\end{array}\right)$ in the basis $e_{1}^{*}, e_{2}^{*}$. In that coordinate system, the symplectic form reads $\omega_{\mathcal{L}}=\operatorname{Tr} d g_{p} \wedge d g_{q}=d p_{1} \wedge d q_{1}+2 d p_{2} \wedge d q_{2}+d p_{3} \wedge d q_{3}$.

The map $u_{p, q}=\Lambda^{-1}\left(L_{p}, g_{q}\right)$ is defined by sending $e_{1}$ and $e_{2}$ to 0 and $e_{3}, e_{4}$ to any basis $v_{1}^{q}, v_{2}^{q}$ of $\mathbb{R}^{2}$ whose Gram matrix is $g_{q}$. Explicitly one has

$$
u_{p, q}\left(a_{1}\right)=-p_{1} v_{1}^{q}-p_{2} v_{2}^{q}, u_{p, q}\left(a_{2}\right)=-p_{2} v_{1}^{q}-p_{3} v_{2}^{q}, u_{p, q}\left(b_{1}\right)=v_{1}^{q}, u_{p, q}\left(b_{2}\right)=v_{2}^{q} .
$$


Writing as a vector the values taken on the symplectic basis we get at $(0,0,0,1,0,1)$ the following derivatives:

$$
\frac{\partial \Lambda^{-1}}{\partial p_{1}}=(-1,0,0,0), \frac{\partial \Lambda^{-1}}{\partial p_{2}}=(-i,-1,0,0), \frac{\partial \Lambda^{-1}}{\partial p_{3}}=(0,-i, 0,0) .
$$

Using the formulas $\frac{\partial v_{1}^{q}}{\partial q_{1}}=\frac{1}{2}, \frac{\partial v_{1}^{q}}{\partial q_{2}}=\frac{\partial v_{1}^{q}}{\partial q_{3}}=0$ and $\frac{\partial v_{2}^{q}}{\partial q_{1}}=0, \frac{\partial v_{2}^{q}}{\partial q_{2}}=1, \frac{\partial v_{2}^{q}}{\partial q_{3}}=\frac{i}{2}$ we get

$$
\frac{\partial \Lambda^{-1}}{\partial q_{1}}=\left(0,0, \frac{1}{2}, 0\right), \frac{\partial \Lambda^{-1}}{\partial q_{2}}=(0,0,0,1), \frac{\partial \Lambda^{-1}}{\partial q_{3}}=\left(0,0,0, \frac{i}{2}\right) .
$$

On the other hand, the symplectic structure on $V$ reads

$$
\omega_{V}(v, w)=-\left\langle v\left(a_{1}\right), w\left(b_{1}\right)\right\rangle+\left\langle w\left(a_{1}\right), v\left(b_{1}\right)\right\rangle-\left\langle v\left(a_{2}\right), w\left(b_{2}\right)\right\rangle+\left\langle w\left(a_{2}\right), v\left(b_{2}\right)\right\rangle .
$$

By checking in the basis, we find $\Lambda^{*} \omega_{V}=\frac{1}{2} \omega_{\mathcal{L}}$ as asserted.

\subsection{Topology of hourglasses.}

Proposition 4.7. The space $X_{0}$ is homeomorphic to $X_{\mathcal{E}}$. The homeomorphism maps the configuration $\left[\rho_{0}\right]$ to 0 , and $C^{\times} / S^{1}$ to $X_{0}^{\times}$. In particular we have:

- $X_{0}^{*}$ is connected, simply connected and satisfies $\pi_{2}\left(X_{0}^{*}\right)=\mathbb{Z}$.

- $X_{0}^{\times}$is homotopically equivalent to $\mathcal{L}$. Hence it is connected and satisfies $\pi_{1}\left(X_{0}^{\times}\right)=$ $\pi_{2}\left(X_{0}^{\times}\right)=\mathbb{Z}$.

Proof. By Proposition 4.2, a punctured neighbourhood of $\rho_{0}$ is diffeomorphic to $X_{\mathcal{E}}^{*}$. As we saw in Section 3.1. $X_{0}^{*}$ is diffeomorphic to the set $f^{-1}(1,1+\varepsilon)$ for any $\varepsilon$ and the result follows. The Hermitian form $h$ has signature $(2,2)$ hence in some coordinates one has $q\left(z_{1}, w_{1}, z_{2}, w_{2}\right)=$ $\left|z_{1}\right|^{2}+\left|z_{2}\right|^{2}-\left|w_{1}\right|^{2}-\left|w_{2}\right|^{2}$. Normalizing the non-zero vectors $\left(z_{1}, z_{2}\right)$ and $\left(w_{1}, w_{2}\right)$ we get the homeomorphism $C^{*} \simeq S^{3} \times S^{3} \times \mathbb{R}$ and hence the homotopy equivalence $X_{0}^{*} \simeq S^{3} \times S^{3} / S^{1}$. The long exact sequence of the fibration $S^{3} \times S^{3} \rightarrow\left(S^{3} \times S^{3}\right) / S^{1}$ gives the fundamental groups of $X_{0}^{*}$ claimed in the proposition.

Using the canonical Riemannian metric on $X_{0}^{*}$, the gradient flow of $f$ preserves the set of sextuples which are on the same line. Hence the same argument as above works for the space $X_{0}^{\times}$which is homeomorphic to $C^{\times} / S^{1}$. This latter space is homeomorphic to $T_{+}^{*} \mathcal{L}$ which is a fiber bundle over $\mathcal{L}$ with convex fiber. Finally we have the homotopy equivalence $X_{0}^{\times} \simeq \mathcal{L}$. Considering the two-fold covering of $\mathcal{L}$ consisting of oriented Lagrangians in $H_{1}(\Sigma, \mathbb{R})$, we get a space $\tilde{\mathcal{L}}=U(2) / S O_{2}$, see [14], Section 2.3. The exact sequence of this fibration gives $\pi_{1}(\tilde{\mathcal{L}}) \simeq$ $\pi_{2}(\tilde{\mathcal{L}}) \simeq \mathbb{Z}$ and the same is true for $\mathcal{L}$.

\section{DYNAMiCS}

5.1. The strategy. As in all proofs of ergodicity of mapping group actions on representation spaces, we will use the periodicity properties of Goldman twist flows. These flows are particularly simple to describe in the framework of sextuples. Let $z=\left(x_{1}, \ldots, x_{6}\right) \in \operatorname{Sex}_{0}$ be a sextuple and suppose that $s_{3} s_{2} s_{1}$ is an elliptic element, that is a rotation over a point $y$. Denote by $R_{t}$ the rotation of angle $t$ over $y$ and pick $\theta$ such that $s_{3} s_{2} s_{1}=R_{\theta}$. Then the formula

$$
\Phi_{123}^{t} z=\left(R_{t} x_{1}, R_{t} x_{2}, R_{t} x_{3}, x_{4}, x_{5}, x_{6}\right)
$$

defines a $2 \pi$-periodic flow on $X_{0}$ such that $\Phi_{123}^{\theta}$ is the half-twist around the first three points. A $\operatorname{Mod}\left(S_{o}\right)$-invariant function on $X_{0}$ is almost everywhere constant along the flow as the angle $\theta$ is almost everywhere irrational. However this argument works only when $s_{3} s_{2} s_{1}$ is elliptic.

All such flows are indexed by partition curves, that is simple curves $\gamma$ in $S_{p}$, the sphere minus the six marked points, which divide the set of points into two subsets of cardinality 3 . Indeed, 
for any $[\rho] \in X_{0}\left(\Gamma_{o}\right)$ we set $\Theta_{\gamma}([\rho]) \in \mathbb{R} / 2 \pi \mathbb{Z}$ to be the rotation number of $\rho(\gamma)$ (that is its angle if it is a rotation and 0 otherwise). Where $\Theta_{\gamma}$ is smooth, we define $X_{\gamma}$ to be the symplectic gradient of $\Theta_{\gamma}$ and extend it by 0 where it is not defined. We denote by $\Phi_{\gamma}^{t}$ the flow of $X_{\gamma}$. This definition is coherent in the sense that $\Phi_{123}=\Phi_{\gamma}$ for a standard partition curve enclosing the 3 first points.

Remark 5.1. Let $\tilde{\gamma}$ be the preimage of $\gamma$ in the surface $\Sigma$ : then $\Phi_{\gamma}^{t}$ is the Goldman flow on $X(\Sigma)$ associated to the separating curve $\tilde{\gamma}$ provided that $\rho(\tilde{\gamma})$ is elliptic.

Definition 5.1. For any $z \in X_{0}$ we set

$$
\mathcal{D}_{z}=\operatorname{Span}\left\{X_{\gamma}(z), \gamma \text { partition curve }\right\} \subset T_{z} X_{0} .
$$

The aim of this section is to prove the following proposition:

Proposition 5.1. The distribution $\mathcal{D}$ is completely non-integrable on the subset $U \subset X_{0}$ of non-pinched configurations.

By construction, the distribution is $\operatorname{Mod}\left(\Gamma_{o}\right)$-invariant, and by Theorem 1.1, the $\operatorname{Mod}\left(\Gamma_{o}\right)$ orbit of any point in $U$ is adherent to the singular representation $\left[\rho_{0}\right]$. Hence it is sufficient to prove Proposition 5.1 by replacing $U$ with any neighbourhood of $\left[\rho_{0}\right]$. This will be a significant simplification for two reasons:

(1) For any partition curve $\gamma, \rho(\gamma)$ is elliptic for $\rho$ close enough to $\rho_{0}$, hence the vector fields $X_{\gamma}$ will not vanish close to the singular representation.

(2) Being completely non-integrable is an open condition, hence, we can replace the functions $\Theta_{\gamma}$ by their Taylor expansion around $\left[\rho_{0}\right]$ and reduce the problem to (symplectic) linear algebra.

The proof of Proposition 5.1 is decomposed into two subsections: in Subsection 5.2 we compute the Taylor expansion of the trace function associated to a partition curve and in Subsection 5.3 , we show that the derivatives of these trace functions generate the cotangent space around $\left[\rho_{0}\right]$.

To conclude this subsection, we recall the argument showing that Proposition 5.1 implies the ergodicity of $\operatorname{Mod}\left(\Gamma_{o}\right)$ on $X_{0}$. Let $f: X_{0} \rightarrow \mathbb{R}$ be a measurable invariant function. Using standard ergodicity arguments (see Proposition 5.4 in [9]), for any partition curve $\gamma$, there is a measure 0 subset $\mathcal{N}_{\gamma} \subset X_{0} \times \mathbb{R}$ such that $f\left(\Phi_{\gamma}^{t}(z)\right)=f(z)$ for all $(z, t) \notin \mathcal{N}_{\gamma}$. Using Fubini theorem and the fact that the flows preserve nullsets, for any partition curves $\gamma_{1}, \ldots, \gamma_{n}$, we will have $f \circ \Phi_{\gamma_{1}}^{t_{1}} \circ \cdots \Phi_{\gamma_{n}}^{t_{n}}(z)=f(z)$ for almost all $\left(z, t_{1}, \ldots, t_{n}\right) \in X_{0} \times \mathbb{R}^{n}$.

Using cutoff functions one can smoothen the vector fields $X_{\gamma}$ without changing the distribution $\mathcal{D}$ - hence we suppose that the vector fields are smooth from now. Let $z \in X_{0}$ be a point in $U$. By the orbit theorem (see [11, Theorem 1 p.33), the orbit $N$ of $z$ through the action of the flows of $X_{\gamma}$ is a submanifold. By Proposition 5.1, the vector fields $X_{\gamma}$ and their brackets evaluated at $z$ generate $T_{z} X_{0}$. Hence $N$ is an open subset of $X_{0}$. Moreover the proof of the orbit theorem in [11] shows that for any $z^{\prime} \in N$ there exist $n \in \mathbb{N}$, curves $\gamma_{1}, \ldots, \gamma_{n}$ and $\left(t_{1}^{0}, \ldots, t_{n}^{0}\right) \in \mathbb{R}^{n}$ such that the map $F: \mathbb{R}^{n} \rightarrow N$ defined by

$$
F\left(t_{1}, \ldots, t_{n}\right)=\Phi_{\gamma_{1}}^{t_{1}} \circ \cdots \circ \Phi_{\gamma_{n}}^{t_{n}}(z)
$$

satisfies $F\left(t_{1}^{0}, \ldots, t_{n}^{0}\right)=z^{\prime}$ and $\operatorname{rank} D F\left(t_{1}^{0}, \ldots, t_{n}^{0}\right)=\operatorname{dim} N=6$. From the fact that $f$ is almost constant in the image of $F$, we get that $f$ is almost everywhere constant in a neighborhood of $z^{\prime}$, hence in $N$, a neighborhood of $z$.

By Proposition 5.1, this argument works for any point in the connected set $U$ which has full measure, showing that $f$ is almost everywhere constant. 
Remark 5.2. In the spirit of Section 3 we can imagine a proof by hand of the transitivity of the flows $\Phi_{\gamma}^{t}$. This proof of Theorem 1.2 would be slightly more direct but less informative about the structure of these hourglass representations, and we chose not to develop it here.

\subsection{Taylor expansion of trace functions. The set}

$$
\widetilde{\mathrm{Sex}}=\left\{\rho: \pi_{1}\left(S_{p}\right) \rightarrow \mathrm{SL}_{2}(\mathbb{R}) \text { such that } \operatorname{Tr} \rho\left(c_{i}\right)=0 \text { for } i=1, \ldots, 6\right\} .
$$

yields a regular covering of $X_{0}$, which is contractible as we proved in Section 3.1. Thus, we may choose once for all a lift $\tilde{\rho_{0}}$ of the singular representation, and lift every representation $\rho$ accordingly. With this setting, for any partition curve $\gamma$, we set $F_{\gamma}([\rho])=\operatorname{Tr} \tilde{\rho}(\gamma)$. This is a continuous function on $X_{0}$, smooth on $X_{0}^{\times}$and which vanishes at $\left[\rho_{0}\right]$. Our purpose is to compute its Taylor expansion at $\left[\rho_{0}\right]$.

Proposition 5.2. For any partition curve $\gamma \subset S_{p}$, let $\tilde{\gamma} \subset \Sigma$ be its (separating) pre-image in $\Sigma$. Then $\Sigma$ can be written as $\Sigma^{\prime} \cup \tilde{\gamma} \Sigma^{\prime \prime}$. Write $\xi \in C$ as $\xi=\xi^{\prime}+\xi^{\prime \prime}$ using the decomposition $H^{1}(\Sigma, \mathbb{C})=H^{1}\left(\Sigma^{\prime}, \mathbb{C}\right) \oplus H^{1}\left(\Sigma^{\prime \prime}, \mathbb{C}\right)$ and set $q_{\gamma}(\xi)=q\left(\xi^{\prime}\right)$, then in the chart given by Proposition 4.2 we have

$$
F_{\gamma}(\xi)= \pm 8 q_{\gamma}(\xi)+o\left(|\xi|^{2}\right) .
$$

Proof. Remark that as $\xi$ is in $C$, we have $q(\xi)=q\left(\xi^{\prime}\right)+q\left(\xi^{\prime \prime}\right)=0$, hence we can replace $\xi^{\prime}$ with $\xi^{\prime \prime}$ in this formula. Up to the action of $\operatorname{Mod}(\Sigma)$ we can suppose that $\gamma=c_{1} c_{2} c_{3}$. The same direct computation as in the proof of Proposition 4.2 then gives

$$
\begin{aligned}
\operatorname{Tr} \tilde{\rho}\left(c_{1}\right) \tilde{\rho}\left(c_{2}\right) \tilde{\rho}\left(c_{3}\right) & = \pm \operatorname{Tr}\left(e^{\xi_{1}} e^{-\xi_{2}} e^{\xi_{3}} s_{0}\right)= \pm \frac{1}{2} \operatorname{Tr} s_{0}\left(\left[\xi_{1}, \xi_{3}\right]-\left[\xi_{1}, \xi_{2}\right]-\left[\xi_{2}, \xi_{3}\right]\right)+o\left(|\xi|^{2}\right) \\
& = \pm \sum_{1 \leq j<k \leq 3}(-1)^{j+k}\left(z_{j} \bar{z}_{k}-z_{k} \bar{z}_{j}\right)+o\left(|\xi|^{2}\right)= \pm 8 q\left(z_{1}, z_{2}, z_{3}, 0,0,0\right)+o\left(|\xi|^{2}\right),
\end{aligned}
$$

with the same notation.

The computation here relates the splitting of $q$ into two terms with the orthogonal decomposition of the cohomology space. We already saw this splitting in the first definition of $X_{\mathcal{E}}$ where the quadratic constraint appeared as a sum of two areas.

5.3. Generating the cotangent space. Let us go back to the proof of Proposition 5.1. We recall that it amounts to proving that the Hamiltonian vector fields $X_{\gamma}$ of the functions $F_{\gamma}$ generate a completely non-integrable distribution close enough to the singular configuration. Using Proposition 5.2 and the fact that being completely non-integrable is an open condition, it reduces to proving the following proposition:

Proposition 5.3. For any partition curve $\gamma$, let $Y_{\gamma}$ be the Hamiltonian vector field of the function $q_{\gamma}$. For any $z \in X_{\mathcal{E}}^{\times}$, set

$$
E_{z}=\operatorname{Span}\left\{Y_{\gamma}(z), \gamma \text { partition curve }\right\} \subset T_{z} X_{\mathcal{E}}^{\times} .
$$

Then, $E$ is a completely non-integrable distribution on $X_{\mathcal{E}}^{\times}$.

Proof. First, we recall that through the symplectic isomorphism $T X_{\mathcal{E}}^{\times} \simeq T^{*} X_{\mathcal{E}}^{\times}$, the vector field $Y_{\gamma}$ corresponds to the covector $d q_{\gamma}$. Hence, the symplectic orthogonal $E^{\omega}$ to the distribution $E$ has the following description:

$$
E_{z}^{\omega}=\left\{w \in T_{z} X_{\mathcal{E}}^{\times}, D_{z} q_{\gamma}(w)=0, \forall \gamma \text { partition curve in } S_{p}\right\} .
$$

We will use the model $X_{\mathcal{E}}^{\times}=C^{\times} / S^{1}$ where $C^{\times}$is the set of surjective maps $v: H_{1}(\Sigma, \mathbb{R}) \rightarrow \mathbb{C}$ such that $q(v)=h(v, v)=0$, with $h(v, w)=-\frac{i}{4} v \cdot \bar{w}$. Recall that a vector $w$ is tangent to $C$ at $v \neq 0$ if $\operatorname{Re} h(v, w)=0$. 
Lemma 5.4. Let $v \in C^{\times}$and let $w \in V$ be tangent to $C$ at $v$ and satisfy $D q_{\gamma}(v)(w)=0$ for any partition curve $\gamma$. Then $v$ and $w$, as linear maps from $H_{1}(\Sigma, \mathbb{R})$ to $\mathbb{R}^{2}$, satisfy the following equation:

$$
\forall x, y \in H_{1}(\Sigma, \mathbb{R}), \quad \operatorname{det}(v(x), w(y))=\operatorname{det}(v(y), w(x)) .
$$

Proof. First, observe that for any symplectic basis $a_{1}, b_{1}, a_{2}, b_{2}$ of $H_{1}(\Sigma, \mathbb{R})$ we have

$$
-4 \operatorname{Re} h(v, w)=\operatorname{det}\left(v\left(a_{1}\right), w\left(b_{1}\right)\right)-\operatorname{det}\left(v\left(b_{1}\right), w\left(a_{1}\right)\right)+\operatorname{det}\left(v\left(a_{2}\right), w\left(b_{2}\right)\right)-\operatorname{det}\left(v\left(b_{2}\right), w\left(a_{2}\right)\right) .
$$

Now, for any partition curve $\gamma$, let $p_{\gamma} \in \operatorname{End}\left(H^{1}(\Sigma, \mathbb{C})\right)$ be the ( $h$-orthogonal) projection on $H^{1}\left(\Sigma^{\prime}, \mathbb{C}\right)$ parallel to $H^{1}\left(\Sigma^{\prime \prime}, \mathbb{C}\right)$; note that $\operatorname{Re} h\left(p_{\gamma}(\cdot), \cdot\right)$ is a symmetric bilinear form, associated to $q_{\gamma}$. Hence the condition $D q_{\gamma}(v)(w)$ is equivalent to $\operatorname{Re} h\left(p_{\gamma}(v), w\right)=0$. If we choose $x=a_{1}$ and $y=b_{1}$ this condition is equivalent to Equation (4).

The same holds if we replace $(x, y)$ with its image by any transformation in $\operatorname{Sp}(4, \mathbb{Z})$. So the $\operatorname{map} \operatorname{Sp}(4, \mathbb{R}) \rightarrow \mathbb{R}$ sending $g$ to $\operatorname{det}(v(g x), w(g y))-\operatorname{det}(v(g y), w(g x))$ vanishes on $\operatorname{Sp}(4, \mathbb{Z})$. By the Zariski-density of $\operatorname{Sp}(4, \mathbb{Z})$ in $\operatorname{Sp}(4, \mathbb{R})$, this forces Equation (4) to hold for any $x, y \in H_{1}(\Sigma, \mathbb{R})$ such that $x \cdot y=1$. By scaling $x$ or $y$, this holds finally for any $x$ and $y$ and the lemma is proved.

Fix $v: H_{1}(\Sigma, \mathbb{R}) \rightarrow \mathbb{C}$ surjective and satisfying $q(v)=0$. Recall from Subsection 4.4 that its kernel $L$ has to be Lagrangian. Let $w$ be in $E_{v}^{\omega}$ : Lemma 5.4 implies that $w$ vanishes on $L$. Hence, $f=w \circ v^{-1}$ is a well defined endomorphism of $\mathbb{R}^{2}$ and writing $x=v^{-1}\left(x^{\prime}\right)$ and $y=v^{-1}\left(y^{\prime}\right)$ we get $\operatorname{det}\left(f\left(x^{\prime}\right), y^{\prime}\right)+\operatorname{det}\left(x^{\prime}, f\left(y^{\prime}\right)\right)=0$. Hence, $f$ preserves infinitesimally the form det, or equivalently has vanishing trace. On the other hand, the equation $\operatorname{Re} h(v, w)=0$ is automatically satisfied in the preceding conditions. To sum up we have shown that the orthogonal distribution $E_{v}^{\omega}$ is the following 2-dimensional space:

$$
E_{v}^{\omega}=\left\{w \in \operatorname{Hom}\left(H_{1}(\Sigma, \mathbb{R}), \mathbb{C}\right),\left.w\right|_{\operatorname{ker} v}=0, \operatorname{Tr}\left(w \circ v^{-1}\right)=0\right\} / \mathbb{R} i v .
$$

If $u \in \operatorname{Hom}\left(H_{1}(\Sigma, \mathbb{R}), \mathbb{C}\right)$ satisfies $\left.u\right|_{L}=0$ then $\operatorname{Re} h(u, v)=0$. All such maps form a Lagrangian containing $E_{v}^{\omega}$. It follows that $E_{v}$ contains this 3-dimensional space. This implies that one can express that $u$ belongs to $E_{v}$ by looking at the restriction of $u$ to $L$.

Let us show that one has the following description:

$$
E_{v}=\left\{u \in \operatorname{Hom}\left(H_{1}(\Sigma, \mathbb{R}), \mathbb{C}\right) \text { s.t. } \exists \lambda \in \mathbb{R},\left.u\right|_{L}=\lambda\left(v^{*}\right)^{-1}\right\} .
$$

In this formula, we see $v$ as a map $H_{1}(\Sigma, \mathbb{R}) / L \rightarrow \mathbb{C}$ and identify $H_{1}(\Sigma, \mathbb{R}) / L$ with $L^{*}$ via the intersection form. Consider an adapted symplectic basis $a_{1}, b_{1}, a_{2}, b_{2}$ of $H_{1}(\Sigma, \mathbb{R})$. An element $w$ in $E_{v}^{\omega}$ vanishes on $a_{1}, a_{2}$ and its matrix $M=\left(w\left(b_{1}\right), w\left(b_{2}\right)\right)$ has trace 0 . Let $u$ be in $E_{v}$ and set $N=\left(u\left(a_{1}\right), u\left(a_{2}\right)\right)$. From the expression of symplectic structure given by equation (3), we derive that the equation $\operatorname{Tr} M N=0$ should be satisfied for all $M$ with 0 trace. Hence $N$ is a scalar matrix and the formula is proved.

This defines a 4-dimensional distribution on $C^{\times} / S^{1}$. It remains to show that it is completely non-integrable which is the content of the last lemma.

Lemma 5.5. Let $\mathcal{L}$ be the Grassmanian of all Lagrangian subspaces of $H_{1}(\Sigma, \mathbb{R})$. Recall the identification $X_{\mathcal{E}}^{\times}: C^{\times} / S^{1}=T_{+}^{*} \mathcal{L}$ and denote by $\pi: T^{*} \mathcal{L} \rightarrow \mathcal{L}$ the standard projection.

Consider the exact sequence

$$
0 \rightarrow T_{L}^{*} \mathcal{L} \rightarrow T_{\left(L, g^{-1}\right)} T^{*} \mathcal{L} \stackrel{d \pi}{\rightarrow} T_{L} \mathcal{L} \rightarrow 0 .
$$

The distribution $E$ corresponds to the distribution $F_{\left(L, g^{-1}\right)}=d \pi^{-1}(\mathbb{R} g)$ and is completely nonintegrable. 
Proof. We will show that the flows of the vector fields generated by elements of $E$ act transitively on $T_{+}^{*} \mathcal{L}$. By Frobenius theorem (see Theorem 4 p. 45 in [1]) and using the fact that $T_{+}^{*} \mathcal{L}$ is an homogeneous space, this is equivalent to the complete non-integrability of $E$.

The identification between $E$ and $F$ is a simple transcription of Equation (5): it remains to show that $F$ is completely non-integrable. Let $T_{+} \mathcal{L} \subset T \mathcal{L}$ be the cone of positive directions. As $F$ contains all the directions of the fiber of $\pi$ one can move from $\left(L, g^{-1}\right)$ to all elements of the form $\left(L,\left(g^{\prime}\right)^{-1}\right)$ for $g^{\prime}$ close to $g$. Following the corresponding direction $g^{\prime}$ in $\mathcal{L}$, one can move from $L$ in $\mathcal{L}$ towards the direction of positive quadratic forms, moving again along the fiber of $\pi$, the problem reduces to show that the conical distribution $T_{+} \mathcal{L}$ is completely non-integrable in $\mathcal{L}$ which is clear from the fact that definite (i.e. positive or negative) quadratic forms linearly generate the space of all quadratic forms.

\section{REFERENCES}

[1] B. H. Bowditch. Markoff triples and quasi-Fuchsian groups. Proc. London Math. Soc. (3), 77(3):697-736, 1998.

[2] Peter Buser. Geometry and spectra of compact Riemann surfaces. Modern Birkhäuser Classics. Birkhäuser Boston Inc., Boston, MA, 2010. Reprint of the 1992 edition.

[3] Louis Funar and Julien Marché. The first Johnson subgroups act ergodically on $\mathrm{SU}_{2}$-character varieties. $J$. Differential Geom., 95(3):407-418, 2013.

[4] Daniel Gallo, Michael Kapovich, and Albert Marden. The monodromy groups of Schwarzian equations on closed Riemann surfaces. Ann. of Math. (2), 151(2):625-704, 2000.

[5] J. Gilman and B. Maskit. An algorithm for 2-generator Fuchsian groups. Michigan Math. J., 38(1):13-32, 1991.

[6] Jane Gilman. Two-generator discrete subgroups of PSL(2, R). Mem. Amer. Math. Soc., 117(561):x+204, 1995.

[7] William M. Goldman. The symplectic nature of fundamental groups of surfaces. Adv. in Math., 54(2):200-225, 1984.

[8] William M. Goldman. Topological components of spaces of representations. Invent. Math., 93(3):557-607., 1988.

[9] William M. Goldman and Eugene Z. Xia. Ergodicity of mapping class group actions on SU(2)-character varieties. In Geometry, rigidity, and group actions, Chicago Lectures in Math., pages 591-608. Univ. Chicago Press, Chicago, IL, 2011.

[10] Michael Heusener and Joan Porti. The variety of characters in $\mathrm{PSL}_{2}(\mathbb{C})$. Bol. Soc. Mat. Mexicana (3), 10(Special Issue):221-237, 2004.

[11] Velimir Jurdjevic. Geometric control theory, volume 52 of Cambridge Studies in Advanced Mathematics. Cambridge University Press, Cambridge, 1997.

[12] Jacques Lafontaine. An Introduction to Differentiable Manifolds. Springer, second edition, 2010.

[13] Julien Marché and Maxime Wolff. The modular action on PSL(2, R)-characters in genus two. Duke Math. Journal, to appear.

[14] Dusa McDuff and Dietmar Salamon. Introduction to symplectic topology. Oxford Mathematical Monographs. The Clarendon Press, Oxford University Press, New York, second edition, 1998.

[15] Joseph P. Previte and Eugene Z. Xia. Topological dynamics on moduli spaces. II. Trans. Amer. Math. Soc., 354(6):2475-2494 (electronic), 2002.

[16] André Weil. Remarks on the cohomology of groups. Ann. of Math. (2), 80:149-157, 1964.

Sorbonne Universités, UPMC Univ. Paris 06, Institut de Mathématiques de Jussieu-Paris Rive Gauche, UMr 7586, CNRS, Univ. Paris Diderot, Sorbonne Paris Cité, 75005 Paris, France

E-mail address: julien.marche@imj-prg.fr

Sorbonne Universités, UPMC Univ. Paris 06, Institut de Mathématiques de Jussieu-Paris Rive

Gauche, UMr 7586, CNRS, Univ. Paris Diderot, Sorbonne Paris Cité, 75005 Paris, France

E-mail address: maxime.wolff@imj-prg.fr 